\title{
A study of open strings ending on giant gravitons, spin chains and integrability.
}

\author{
David Berenstein ${ }^{1, \dagger}$, Diego H. Correa ${ }^{2 \ddagger}$ and Samuel E. Vázquez ${ }^{1, \S}$ \\ 1 Department of Physics, UCSB, Santa Barbara, CA 93106 \\ 2 Centro de Estudios Cientifícos, Valdivia, Casilla 1469, Chile \\ $\dagger$ dberens@physics.ucsb.edu ${ }^{\ddagger}$ dcorrea@cecs.cl \\ $\S$ svazquez@physics.ucsb.edu
}

\begin{abstract}
We systematically study the spectrum of open strings attached to half BPS giant gravitons in the $N=4 \mathrm{SYM}$ AdS/CFT setup. We find that some null trajectories along the giant graviton are actually null geodesics of $A d S_{5} \times S^{5}$, so that we can study the problem in a plane wave limit setup. We also find the description of these states at weak 't Hooft coupling in the dual CFT. We show how the dual description is given by an open spin chain with variable number of sites. We analyze this system in detail and find numerical evidence for integrability. We also discover an interesting instability of long open strings in Ramond-Ramond backgrounds that is characterized by having a continuum spectrum of the string, which is separated from the ground state by a gap. This instability arises from accelerating the Dbrane on which the strings end via the Ramond-Ramond field. From the integrable spin chain point of view, this instability prevents us from formulating the integrable structure in terms of a Bethe Ansatz construction.
\end{abstract}

KEYWORDs: AdS/CFT, integrable spin chains, D-branes. 


\section{Contents}

1. Introduction 2

2. Open Strings on giant gravitons in $A d S_{5} \times S^{5} 6$

2.1 Spherical D3-branes in $A d S_{5} \times S^{5}$

2.2 A Penrose limit for an open string on a non-maximal giant 8

2.3 Open Strings on the pp-wave Geometry (Short Strings) 9

2.4 Semiclassical Open Strings (Long Strings) 12

3. Open Strings on Giant Gravitons from $\mathcal{N}=4$ SYM

3.1 Open Strings as Variable Length Spin Chains 15

3.2 Evidence for a BMN Limit 18

3.3 The Semiclassical Limit 21

4. A D-brane Instability? 23

4.1 One Site 24

4.2 Two Sites 25

4.3 Multiple Sites 27

4.4 A toy model for the D-brane instability 29

5. Evidence for Integrability 32

6. Discussion 33

A. Penrose limit 37

B. Brane in the Penrose limit 39

C. Combinatorics and field theory calculations 40

D. Calculations in the $S U(3)$ sector 46

E. Coherent states for the $q$-deformed algebra 48 


\section{Introduction}

The gauge theory/gravity duality is probably one of the deepest ideas in theoretical physics, in that it gives us in principle the possibility to understand quantum gravity exactly in some setups. The main tool to address this duality involves the ideas of 't Hooft of the large $N$ expansion of field theories in terms of a dual string theory [ []]. A lot of recent progress has happened because there are some examples where the dual string theory is known. The simplest and most studied example of this duality is the original formulation of the AdS/CFT correspondence in terms of maximally supersymmetric Yang Mills theory in four dimensions and type IIB superstring theory compactified on $A d S_{5} \times S^{5}$ [2].

The setup is such that in the limit where $R$ (the radius of curvature of $A d S_{5} \times S^{5}$ ) is large, the field theory is strongly coupled in the sense of 't Hooft: $R^{4} \sim g_{Y M}^{2} N$ is large. Indeed, it would be very nice to have a good understanding of how this duality works in detail, as it would also give us hints on how to calculate observables in strongly coupled gauge theories in four dimensions for more general setups.

There are various avenues to explore this correspondence, based on different observables that one would want to work with. Historically, the first set of observables to be addressed were the dual states to single graviton perturbations of $A d S_{5} \times S^{5}$ [3, [] and how to go about testing their correlation functions. These states are all members of BPS multiplets, so their energies are protected by supersymmetry.

A few years later, it was discovered that there were interesting geometric limits where the string theory could be quantized exactly [5, 6, 7]. Translating the corresponding quantum numbers of states to the field theory language provided a new large $N$ limit where one also scales the energy and $R$ charge of the observables as one makes the coupling constant large [ [ 8 . The subsector of states that one focuses on is closely tied to the supersymmetry of the original system, so the states in questions are nearly supersymmetric. In these cases, perturbation theory at strong coupling was parametrically suppressed by the large quantum numbers of the states in question, so it was possible to use perturbation theory to examine the strong coupling regime of the field theory in a relatively safe environment.

This result produced an interest to make a systematic study of perturbation theory near the free field limit. In particular, it became interesting to compute the full spectrum of anomalous dimensions of all local operators on the field theory.

In the case where one focuses on operators whose free field dimension grows

at most as $\sqrt{N}$, the states are roughly described by a Fock space of closed string states, and the dimension of the operators in the large $N$ limit is dominated by the planar diagram expansion. The expansion to one loop order of this problem revealed a very surprising structure. Minahan and Zarembo [9] discovered that the one loop spectrum of anomalous dimensions in a subsector of the theory gave rise to an integrable $S O(6)$ spin chain: a generalization of the Heisenberg $X X X$ spin 
chain. This result was later generalized to include the full set of local operators of the theory and it was shown that the full planar one loop spectrum of anomalous dimensions gave rise to an integrable spin chain model that could be solved by Bethe Ansatz techniques [10]. In a parallel development, Bena et. al. [11] discovered that the string sigma model on $A d S_{5} \times S^{5}$ was also integrable giving rise to the idea that the integrability structure of the string on $A d S_{5} \times S^{5}$ could be used as a vehicle to understand the AdS/CFT correspondence in detail, at least in the maximally supersymmetric case [12]. However, integrability is not a requisite of the AdS/CFT correspondence, so apart from circumstantial evidence for integrability at weak and strong coupling it is not clear that this will be the final answer to the AdS/CFT puzzle.

Obviously, having an integrable structure is a strong constraint on the string dynamics and it has been shown that many interesting general perturbations of the maximally supersymmetric background destroy the integrability properties (see [13] for example). In a similar vein, integrability can also happen for spin chain models with boundaries and it is interesting to examine if such integrable structures can also appear in the perturbative Yang Mills theory and use them as a tool to examine other configurations of matter in the AdS/CFT correspondence. Indeed, the natural boundaries for open strings are D-branes, so one can try to see if D-brane solutions preserve the integrability of the string theory and then try to solve the string spectrum to understand the D-branes, much like solving the string spectrum should help to understand gravity.

Here, one can envision two different types of D-branes. The first corresponds to adding defects to the field theory so that there are "flavor branes" of various dimensionalities, and the quarks can act as boundaries for the spin-chain string. These branes are always infinite. This has been explored in [14, 15, 16, with the result that the one-loop set of anomalous dimensions are actually integrable.

A second approach is to study finite volume D-branes on the AdS geometry. These correspond to non-perturbative states of finite energy in the string description, so one can try to find the dual description of these states in the field theory. Because of issues of strong coupling physics, it is better if the corresponding states are supersymmetric to have some protection for the calculations. From this point of view, one has to look for supersymmetric D-brane configurations in the AdS geometry, and to their corresponding dual description in the field theory. Once these D-branes are found, one should be able to describe the open strings attached to them and study if these strings can be described by a boundary spin chain model. After this is done, one can ask if the model corresponds to some form of integrability or not and if this integrability is of the familiar Bethe-Ansatz form or not. A lot more care is needed in this case because the branes are finite. This finiteness implies that the D-brane can back-react to the presence of the string and the dynamics can be much more complicated than in the case of an infinite brane. 
This is the problem that we will concern ourselves in this paper. The branes under question are going to be giant gravitons [17]. These preserve half of the supersymmetries and their dual field theory states are known very well [18, 19, 20]. How to add strings to giants was discussed in the works [21, 22, 23, 24] which also included a description of how the enhanced gauge symmetry of coinciding D-branes could be understood. One of the main difficulties in performing the calculations is that the dimension of the operator is of order $N$ and it is harder to separate the planar and non-planar contributions. The dimension of the operator implies that there is a combinatorial enhancement of the usual $1 / N$ suppressions, so it is not clear a priori that there is a well defined procedure that works in the general case, and one has to work example by example to understand the dynamics of the purported D-brane state.

The one loop spectrum of anomalous dimensions for strings attached to a maximal giant graviton was described in [25], were it was found that the one loop planar anomalous dimensions correspond to an ordinary spin chain model with integrable Dirichlet-like boundary conditions. This work was extended to study what spin chain corresponds to a more general giant graviton in [26], where we found that the spin chain in question has a variable number of sites and therefore it is not an ordinary spin chain model anymore. After a bosonization transformation, we found that the spin chain model could be also understood in terms of a system of a Cuntz oscillator chain model (a boson chain, where each spin corresponds to a single boson Fock space) with non-diagonal boundary conditions. The non-diagonal boundary conditions imply that the total boson number is not conserved. Our setup is also the limit $q \rightarrow 0$ of a $q$-boson chain model with non-diagonal boundary conditions, where the $q$ boson is defined by the algebra $a a^{\dagger}-q a^{\dagger} a=1$, with $q$ a real number. More recently, it has been claimed by [27] that in the maximal giant graviton case, there seems to be a problem with integrability beyond the two loop order, in contrast to the closed spin chain model [29, 30]. Agarwal argued that the Bethe Ansatz breaks down by direct calculation at two loops and therefore the model is not integrable. Another claim that studies the consistency of a Bethe Ansatz for the maximal giant graviton by Okamura and Yoshida suggest that the BMN limit breaks down instead [28]. We have a different interpretation of these facts: if the system is integrable, it will not realize integrability by a Bethe Ansatz. To do this, we study more general giant gravitons.

In this paper we extend our analysis of 26] to try to understand the full spectrum of the variable length spin chain model. The hamiltonian of the spin chain model is given by

$$
\begin{aligned}
H= & 2 \lambda \sum_{l=1}^{L} \hat{a}_{l}^{\dagger} \hat{a}_{l}-\lambda \sum_{l=1}^{L-1}\left(\hat{a}_{l}^{\dagger} \hat{a}_{l+1}+\hat{a}_{l} \hat{a}_{l+1}^{\dagger}\right) \\
& +2 \lambda \alpha^{2}+\lambda \alpha\left(\hat{a}_{1}^{\dagger}+\hat{a}_{1}\right)+\lambda \alpha\left(\hat{a}_{L}^{\dagger}+\hat{a}_{L}\right),
\end{aligned}
$$


and the operators $\hat{a}_{i}$ obey the Cuntz algebra for a single species,

$$
\hat{a}_{i} \hat{a}_{i}^{\dagger}=I, \quad \hat{a}_{i}^{\dagger} \hat{a}_{i}=I-|0\rangle\langle 0|
$$

where operators corresponding to different sites commute. ${ }^{1}$

In our exploration of the model we will find very interesting phenomena that at first sight seem to contradict a very naive intuition about the system. The naive intuition is that the open string ending on the D-brane has a discrete spectrum, because one has a finite energy configuration of matter in the $A d S_{5}$ geometry. However, there is a technical issue with the fact that the energy is going to infinity as we take $N$ large, because the mass of the D-brane scales with $N$. The naive answer, in spite of being technically correct, might also include some non-perturbative information on the finiteness of $N$, that is not necessarily reflected in the strict large $N$ limit. Indeed, we find that the spectrum of the spin chain model is continuous. Upon thinking further about it, it becomes obvious that the spin chain answer correlates very well with the AdS geometric intuition and we will explain how this puzzle is solved in detail.

The continuous string spectrum also has some other consequences for the integrability program. It seems to indicate that if integrability is present, it will not be of the form of a Bethe ansatz solution. From this point of view, if integrability is present, the conjectures in [30] need to be extended appropriately, or it is even possible that they might need to be revised completely. For finite size systems, a Bethe ansatz solution would predict that the spectrum of the system is related to solutions (roots) of polynomial equations of high degree, giving rise to a discrete spectrum of configurations. Does this fact destroy the integrability of the open string? We don't think so. Although it is hard to come by with integrable systems that can not be solved by Bethe Ansatz, there are some well known examples of integrable systems that manage to be integrable without having a Bethe ansatz solution. Indeed, the $c=1$ matrix model is such an example. This model is dual to a two dimensional string in a linear dilaton background. It is very well known that the number of particle excitations is not conserved when scattering of the Liouville wall, so this non-conservation of the number of particle excitations is contrary to the typical Bethe ansatz solution. There are other hints that a Bethe ansatz is not the complete story for describing the individual string dynamics at strong 't Hooft coupling [31]. We believe our results in this paper show that the Bethe ansatz breaks down perturbatively a lot faster for the open string than for the closed string.

Our paper is organized as follows. We begin in section 2 by reviewing the physics of giant gravitons. We show how the local geometry near an open string spinning

\footnotetext{
${ }^{1}$ Since spin chain models also have applications to other areas of physics, the reader who is only interested in the analysis of the spin chain model can jump to section 3 and should also read sections 田它
} 
around the moving giant graviton arises from a Penrose limit of $A d S_{5} \times S^{5}$. We quantize some of the bosonic modes of the open string in that background, which is valid for short strings that sense the local geometry. We then consider longer strings that carry two angular momenta, one of them in the same direction as the giant graviton. Then we expand the Polyakov action in the limit of large transverse angular momentum just like with closed strings [32, 34].

In section 3 we move to the dual description in SYM theory. We review the derivation of the matrix of anomalous dimensions interpreted as the Hamiltonian of a spin chain with variable number of sites. We then map this problem to a lattice of bosons with sources and sinks at the boundaries. Then, we show numerical evidence for the agreement of the bosonic Hamiltonian spectrum with the plane wave spectrum of the dual open string. We also use coherent states to obtain a sigma model action in the limit of large R-charge. It agrees with the Polyakov action of the open string in the large momentum limit, in a particular gauge. Moreover we show how the SYM theory gives the correct boundary conditions for the open strings.

In section 1 we show that the spectrum of the anomalous dimension matrix contains continuum energy bands. We then argue that these can represent an instability of the dual D-brane where the open string can absorb a significant fraction of the angular momentum of the giant, making it collapse. In fact, we show that the lowest energy band is accessible to semiclassical string excitations.

In section 5 we show some numerical evidence that lead us to conjecture the integrability of the Hamiltonian. Finally, in section 6, we discuss our results and a list of open problems that hopefully will be of interest for the reader. Some of the details of the calculations for the different sections are shown in the appendices.

\section{Open Strings on giant gravitons in $A d S_{5} \times S^{5}$}

In this section we study giant gravitons in $S^{5}$ and their open string excitations from the point of view of string theory/supergravity. In the next section we will see how this stringy description arises from the dual SYM theory.

We begin by briefly reviewing some of the basic geometrical properties of the spherical D3-branes existing in the background of $A d S_{5} \times S^{5}$ that wrap an $S^{3}$ inside the $S^{5}$ [17]. These spherical branes are commonly called giant gravitons.

They are 1/2 BPS and their classical stability is due to the presence of the fiveform flux which exactly cancels their tension. As we will see, the movement of the non-maximal giant gravitons gives rise to non-trivial boundary conditions to open string excitations. We are interested in studying fast moving (nearly supersymmetric) strings on the D-brane (close to the speed of light and near the point particle limit), as for these strings the dynamics simplifies. These should be closely related to null trajectories in $A d S_{5} \times S^{5}$ that are contained in the D-brane worldvolume trajectory. Moreover, as we now show, for the special supersymmetric case the geometry near 
this null trajectory is the usual pp-wave of type IIB supergravity, so we will focus on this case.

\subsection{Spherical D3-branes in $A d S_{5} \times S^{5}$}

Spherical stable branes are known to exist in the maximally supersymmetric background of type IIB supergravity $A d S_{5} \times S^{5}$ [17]. Indeed, these branes are actually supersymmetric. They can wrap an $S^{3}$ either inside the $S^{5}$ or inside of $A d S_{5}$ [35, 36]. In this section and in the rest of the paper we will focus on the former ones.

To begin with, let us write the metric of the $A d S_{5} \times S^{5}$ background as

$$
d s^{2}=R^{2}\left(-\cosh ^{2} \rho d t^{2}+d \rho^{2}+\sinh ^{2} \rho d \Omega^{\prime 2}{ }_{3}^{2}+d \theta^{2}+\cos ^{2} \theta d \psi^{2}+\sin ^{2} \theta d \Omega_{3}^{2}\right),
$$

and the 3-sphere metrics as

$$
\begin{aligned}
& d \Omega_{3}^{2}=d \varphi^{2}+\cos ^{2} \varphi d \eta^{2}+\sin ^{2} \varphi d \xi^{2} \\
& d \Omega_{3}^{\prime 2}=d \varphi^{\prime 2}+\cos ^{2} \varphi^{\prime} d \eta^{\prime 2}+\sin ^{2} \varphi^{\prime} d \xi^{\prime 2}
\end{aligned}
$$

In these coordinates, the Ramond-Ramond 4-form potential takes the form

$$
C^{(4)}=4 \pi N \alpha^{\prime 2}\left(\sinh ^{4} \rho d t \wedge \Omega_{3}^{\prime}-\sin ^{4} \theta d \psi \wedge \Omega_{3}\right)
$$

and we have the relation $R^{4}=4 \pi g_{s} N \alpha^{\prime 2}$.

McGreevy, Sussking and Toumbas found spherical D3-branes carrying angular momentum in the $S^{5}$ [17]. They are supersymmetric solutions of the brane action, that expand in the 3 -sphere $\Omega_{3}$. Choosing the static gauge, the parametric coordinates of the brane $\left(\sigma_{0}, \sigma_{1}, \sigma_{2}, \sigma_{3}\right)$ can be identified with space-time coordinates

$$
t=\sigma_{0}, \quad \varphi=\sigma_{1}, \quad \eta=\sigma_{2}, \quad \xi=\sigma_{3} .
$$

The brane carries angular momentum along the $\psi$ direction and it is located at $\rho=0$ and at a constant $\theta=\theta_{0}$.

$$
\psi=\psi\left(\sigma_{0}\right), \quad \rho=0, \quad \theta=\theta_{0},
$$

The equations of motion are solved with $\dot{\psi}$ constant. More precisely, and independently of $\theta_{0}$, by $\dot{\psi}=1$. Thus, the center of mass of the giant graviton is moving along an equatorial null trajectory. However, each element of the giant is moving in a time-like orbit of radius $r_{e l}=R \cos \theta_{0}$. The radius of the giant also depends on $\theta_{0}$, $r_{g g}=R \sin \theta_{0}$.

For $\dot{\psi}=1$, the momentum conjugate to $\psi$ becomes

$$
p=N \sin ^{2} \theta_{0}
$$


We can use this relation for highlighting a relevant feature of giant gravitons: their radii grows as they increase their angular momenta

$$
r_{g g}=R \sqrt{\frac{p}{N}}
$$

Since we are considering giant gravitons that expand in $S^{5}$, the radius is bounded by $r_{g g} \leq R$ and hence the angular momentum is also bounded by the number of units of five-form flux on the sphere

$$
p \leq N
$$

When the equality is satisfied the brane solution is known as maximal giant graviton. Notice that in this case the radius of the orbit of an element of the giant $r_{e l}=\sqrt{1-p / N}$ shrinks to zero and all the angular momentum comes from the term with the Ramond-Ramond form in the brane action. Being the maximal giant graviton static is the reason why this special case turns out to be simpler. However, considering non-maximal giant gravitons gives rise to interesting and novel phenomena.

\subsection{A Penrose limit for an open string on a non-maximal giant}

We obtain in this section, the effective geometry seen, in the large $N$ limit, by an open string with angular momentum $J$ growing as $\sqrt{N}$, attached to a giant graviton whose angular momentum $p$ is growing proportionally to $N$. Then, the angle $\theta_{0}$ is kept constant and the radius of the giant diverges. The open string will be effectively attached to a flat D3-brane in a pp-wave background. For the maximal giant graviton this was already analyzed in 22]. We now focus on smaller or non-maximal giant gravitons.

As we already said, each element of the giant graviton is moving in a time-like trajectory. In order to travel in null trajectory, an observer on the giant should be spinning fast along it. Then, we will study open strings with two components of angular momenta. In particular, we now consider a trajectory along $\psi$ and $\eta$, keeping $\varphi=0$. A null trajectory should satisfy

$$
R^{2}\left(-\dot{t}^{2}+\cos ^{2} \theta_{0} \dot{\psi}^{2}+\sin ^{2} \theta_{0} \dot{\eta}^{2}\right)=0
$$

Since $\dot{t}=1$ and $\dot{\psi}=1$, one necessarily has $\dot{\eta}= \pm 1$. Although it is not obvious at first sight, this null trajectory is a null geodesic of $A d S_{5} \times S^{5}$ and then a customary Penrose limit can be taken. At this point it is worth highlighting the ratio of the angular momentum in both angular directions

$$
\frac{J_{\psi}}{J_{\eta}}=\cot ^{2} \theta_{0}=\frac{N}{p}\left(1-\frac{p}{N}\right) .
$$

We will later see the natural emergence of this quantity in the gauge theory side. 
By following the standard Penrose limit procedure (see appendix A for details) we get a plane-wave geometry with the metric

$$
d s^{2}=-4 d u d v+4 y d u d x-\sum_{a=1}^{6} z_{a}^{2} d u^{2}+d x^{2}+d y^{2}+\sum_{a=1}^{6} d z_{a}^{2} .
$$

The RR 5-form field strength becomes

$$
F_{(5)}=2 d u \wedge\left(d z_{1} \wedge d z_{2} \wedge d z_{3} \wedge d z_{4}+d z_{5} \wedge d z_{6} \wedge d z_{7} \wedge d z_{8}\right)
$$

This is nothing but the usual maximally supersymmetric pp-wave of type IIB supergravity [5] displayed in unusual coordinates. This can be explicitly seen with an appropriate coordinate transformation [37, 38.

\subsection{Open Strings on the pp-wave Geometry (Short Strings)}

Let us now consider the open string theory in the pp-wave geometry (2.11) corresponding to an open string spinning along a non-maximal giant. This is a good description for short strings which sense the local geometry near the giant graviton. We will focus on the bosonic sector of the superstring and, in particular, on those modes that later will be compared to the gauge theory predictions. More precisely, we will focus on open strings carrying two angular momenta on the sphere with one of them in the direction of movement of the giant graviton.

The bosonic part of the superstring action is

$$
\begin{gathered}
S=-\frac{1}{4 \pi \alpha^{\prime}} \int d \tau \int_{0}^{\pi} d \sigma\left(-4 \partial^{\alpha} u \partial_{\alpha} v+4 y \partial^{\alpha} u \partial_{\alpha} x-z_{i} z_{i} \partial^{\alpha} u \partial_{\alpha} u\right. \\
\left.+\partial^{\alpha} x \partial_{\alpha} x+\partial^{\alpha} y \partial_{\alpha} y+\partial^{\alpha} z_{i} \partial_{\alpha} z_{i}\right) .
\end{gathered}
$$

Fixing the light-cone gauge with the usual procedure

$$
u=2 \alpha^{\prime} p^{u} \tau
$$

we obtain the light-cone action

$$
S_{l c}=-\frac{1}{4 \pi \alpha^{\prime}} \int d \tau \int_{0}^{\pi} d \sigma\left(\partial^{\alpha} x \partial_{\alpha} x+\partial^{\alpha} y \partial_{\alpha} y-4 m y \dot{x}+\partial^{\alpha} z_{i} \partial_{\alpha} z_{i}+m^{2} z_{i} z_{i}\right)
$$

where dots and primes refer to derivatives with respect to $\tau$ and $\sigma$ respectively. We have also defined the mass $m=2 \alpha^{\prime} p^{u}$. As in the case of closed strings [39], this action can also be derived as the quadratic order expansion of the string action around the classical solution $\theta=\theta_{0}$ and $\psi=t$.

The equations of motion from (2.15) are

$$
\begin{aligned}
\left(\partial^{\alpha} \partial_{\alpha}-m^{2}\right) z_{i} & =0, \\
\partial^{\alpha} \partial_{\alpha} x+2 m \dot{y} & =0, \\
\partial^{\alpha} \partial_{\alpha} y-2 m \dot{x} & =0 .
\end{aligned}
$$


For $z_{i}$ we obtain the usual massive equations. For $x$ and $y$ it is convenient to define a complex field $w=x+i y$ which satisfies

$$
\partial^{\alpha} \partial_{\alpha} w-2 i m \dot{w}=0,
$$

A solution of (2.19) is $w=e^{-i m \tau} W$ whenever $W$ is a solution of the massive equation

$$
\left(\partial^{\alpha} \partial_{\alpha}-m^{2}\right) W=0 \text {. }
$$

We now have to specify the boundary conditions for these fields. To this end, we have to keep track of the D-brane position in the Penrose limit. This can be done using the coordinate transformation of the Penrose limit (see appendix B). The original boundary conditions are translated into Neumann boundary conditions for $u, v, z_{1}, z_{2}$ and Dirichlet boundary conditions for $x, y, z_{3}, z_{4}, z_{5}, z_{6}$.

The mode expansion and the canonical quantization of fields $z_{1}, \ldots, z_{6}$ (those satisfying massive equations of motion), goes exactly as in [40]. We concentrate on excitations of fields $x$ and $y$ which later will be related to the dual description.

Solutions of (2.20) satisfying Dirichlet boundary conditions can be expanded as

$$
W=\sum_{n>0} \sin (n \sigma) \sqrt{\frac{4 \alpha^{\prime}}{\omega_{n}}}\left(\beta_{n} e^{-i \omega_{n} \tau}+\tilde{\beta}_{n}^{*} e^{i \omega_{n} \tau}\right),
$$

where $\omega_{n}=+\sqrt{n^{2}+m^{2}}$. The factor $\sqrt{4 \alpha^{\prime} / \omega_{n}}$ in the coefficients is included for later convenience. The expansions for the original fields $x$ and $y$ are

$$
\begin{aligned}
& x=\frac{1}{2} \sum_{n>0} \sin (n \sigma) \sqrt{\frac{4 \alpha^{\prime}}{\omega_{n}}}\left(\tilde{\beta}_{n} e^{-i \omega_{n}^{-} \tau}+\beta_{n} e^{-i \omega_{n}^{+} \tau}+\tilde{\beta}_{n}^{*} e^{i \omega_{n}^{-} \tau}+\beta_{n}^{*} e^{i \omega_{n}^{+} \tau}\right), \\
& y=\frac{i}{2} \sum_{n>0} \sin (n \sigma) \sqrt{\frac{4 \alpha^{\prime}}{\omega_{n}}}\left(\tilde{\beta}_{n} e^{-i \omega_{n}^{-} \tau}-\beta_{n} e^{-i \omega_{n}^{+} \tau}-\tilde{\beta}_{n}^{*} e^{i \omega_{n}^{-} \tau}+\beta_{n}^{*} e^{i \omega_{n}^{+} \tau}\right),
\end{aligned}
$$

where now, $\omega_{n}^{ \pm}=\omega_{n} \pm m$. The string spectrum can be obtained by canonical quantization. With our normalization, the coefficients upgraded to operators satisfy two set of mutually commuting oscillator-like algebra,

$$
\begin{array}{llrl}
{\left[\beta_{n}, \beta_{m}^{\dagger}\right]} & =\delta_{n m}, & & {\left[\beta_{n}, \beta_{m}\right]=\left[\beta_{n}^{\dagger}, \beta_{m}^{\dagger}\right]=0,} \\
{\left[\tilde{\beta}_{n}, \tilde{\beta}_{m}^{\dagger}\right]=\delta_{n m},} & {\left[\tilde{\beta}_{n}, \tilde{\beta}_{m}\right]=\left[\tilde{\beta}_{n}^{\dagger}, \tilde{\beta}_{m}^{\dagger}\right]=0 .}
\end{array}
$$

The spectrum of the sector we are considering is obtained by acting with the creation operators $\beta_{m}^{\dagger}$ and $\tilde{\beta}_{m}^{\dagger}$ on a vacuum state satisfying $\beta_{m}|0\rangle=\tilde{\beta}_{m}|0\rangle=0$. The light-cone Hamiltonian can be expressed in terms of the number operators corresponding to the oscillator operators. Scaling $\tau$ and $\sigma$ by $2 \alpha^{\prime} p^{u}$

$$
\begin{aligned}
H_{l c}^{x y} & =\frac{1}{8 \alpha^{\prime 2} p^{u}} \int_{0}^{2 \pi \alpha^{\prime} p^{u}} d \sigma\left(\dot{x}^{2}+\dot{y}^{2}+x^{\prime 2}+y^{\prime 2}\right) \\
& =\frac{1}{2 \alpha^{\prime} p^{u}} \sum_{n>0}\left(\omega_{n}^{-} \tilde{\beta}_{n}^{\dagger} \tilde{\beta}_{n}+\omega_{n}^{+} \beta_{n}^{\dagger} \beta_{n}\right) .
\end{aligned}
$$


We see that excitations created by $\beta_{n}^{\dagger}$ have more energy than those created by $\tilde{\beta}_{n}^{\dagger}$. Expanding the square roots in $\omega_{n}$

$$
H_{l c}^{x y} \approx \sum_{n>0}\left[\tilde{N}_{n} \frac{n^{2}}{8 \alpha^{\prime 2} p^{u 2}}+N_{n}\left(2+\frac{n^{2}}{8 \alpha^{\prime 2} p^{u 2}}\right)\right],
$$

where $N_{n}=\beta_{n}^{\dagger} \beta_{n}$ and $\tilde{N}_{n}=\tilde{\beta}_{n}^{\dagger} \tilde{\beta}_{n}$.

The Hamiltonian and angular momentum generators are, in the light-cone gauge,

$$
\begin{aligned}
H_{l c} & =-p_{u}=i \frac{\partial}{\partial u}, \\
p^{u} & =-\frac{1}{2} p_{v}=\frac{i}{2} \frac{\partial}{\partial v} .
\end{aligned}
$$

Using the change of coordinates (A.1) we can express them in terms of the original generators

$$
\begin{aligned}
H_{l c} & =i\left(\frac{\partial}{\partial t}+\frac{\partial}{\partial \psi}+\frac{\partial}{\partial \eta}\right)=\Delta-J_{\psi}-J_{\eta} \\
p^{u} & =\frac{i}{2 R^{2}}\left(\frac{\partial}{\partial t}-\frac{\partial}{\partial \psi}-\frac{\partial}{\partial \eta}\right)=\frac{\Delta+J_{\psi}+J_{\eta}}{2 R^{2}}
\end{aligned}
$$

Looking forward to a gauge theory interpretation we can label the angular momenta as (c.f. (2.10) ),

$$
\begin{aligned}
J_{\eta} & =L \\
J_{\psi} & =\cot ^{2} \theta_{0} L=\frac{\alpha^{2}}{1-\alpha^{2}} L .
\end{aligned}
$$

where we define $\alpha \equiv \sqrt{1-p / N}$. Then, the sum of angular momenta is

$$
J_{\eta}+J_{\psi}=\frac{L}{1-\alpha^{2}}
$$

For a finite light-cone energy it is required that $\Delta \simeq L /\left(1-\alpha^{2}\right)$ and then $p^{u} \simeq$ $L / R^{2}\left(1-\alpha^{2}\right)$. Finally, using $R^{4}=4 \pi g_{s} N \alpha^{2}$ and $\lambda=g_{s} N / 2 \pi$, the energy of each excitation is

$$
\tilde{E}_{n} \approx \frac{\lambda \pi^{2}\left(1-\alpha^{2}\right)^{2} n^{2}}{L^{2}}, \quad E_{n} \approx 2+\frac{\lambda \pi^{2}\left(1-\alpha^{2}\right)^{2} n^{2}}{L^{2}},
$$

for $x$ and $y$ respectively. Thus, as expected, we see that string theory predicts a BMN limit for the anomalous dimension of the dual operators describing these open string excitations. We will come back to the dual interpretation of these energies in section 3 . 


\subsection{Semiclassical Open Strings (Long Strings)}

If we want to consider more general open strings ending on the giant graviton, we need to include the full $A d S_{5} \times S^{5}$ background in the Polyakov action. However, it is well known by now that we should expect a classical description in the large angular momentum limit ${ }^{2}$. Again, as we did for short strings in the pp-wave geometry, we focus on the excitation of two coordinates of the string. More precisely, the two coordinates of the sphere $S^{5}$ subject to Dirichlet boundary conditions. The reason is that the description of these excitations in the dual theory is more easily isolated from the rest. Moreover we will fix a particular gauge of reparametrization invariance different from the standard conformal gauge, following closely [34]. This election will be the reflection of the a particular labeling of the operators in the dual gauge theory.

These results were already presented in a previous letter [26]. We reproduce them here providing more details of their derivation. The starting point is the Polyakov action in phase space. We can write the conjugate momenta as,

$$
p_{\mu}=-G_{\mu \nu}\left(A \partial_{0} x^{\mu}+B \partial_{1} x^{\mu}\right),
$$

where $A=\sqrt{-g} g^{00}, B=\sqrt{-g} g^{01}$ and $g^{a b}$ is the worldsheet metric. The Polyakov action then takes the form

$$
S_{p}=\sqrt{\lambda_{Y M}} \int d \tau \int_{0}^{\pi} \frac{d \sigma}{2 \pi} \mathcal{L},
$$

where,

$$
\mathcal{L}=p_{\mu} \partial_{0} x^{\mu}+\frac{1}{2} A^{-1}\left[G^{\mu \nu} p_{\mu} p_{\nu}+G_{\mu \nu} \partial_{1} x^{\mu} \partial_{1} x^{\nu}\right]+B A^{-1} p_{\mu} \partial_{1} x^{\mu} .
$$

Here we have factorized the radius of $A d S_{5}$ and $S^{5}$ so that by the AdS/CFT correspondence $\lambda_{Y M}=g_{Y M}^{2} N=8 \pi^{2} \lambda=R^{4} / \alpha^{\prime 2}$. Moreover, $A, B$ play the role of Lagrange multipliers implementing the constraints $T_{a b}=0$.

For an open string traveling with the giant graviton and with excitations on the sphere only, the effective geometry is $\mathbb{R} \times S^{5}$. We can write the corresponding metric as,

$$
d s^{2}=-d t^{2}+|d X|^{2}+|d Y|^{2}+|d Z|^{2},
$$

where $|X|^{2}+|Y|^{2}+|Z|^{2}=1$. The giant graviton will be orbiting in the $Z$ direction with $Z=\sqrt{1-p / N} e^{i t}$ and will wrap the remaining $S^{3}$. We will put our string at $X=0$. Then we define the coordinates,

$$
\begin{aligned}
Z & =r e^{i(t-\phi)}, \\
Y & = \pm \sqrt{1-r^{2}} e^{i \varphi},
\end{aligned}
$$

\footnotetext{
${ }^{2}$ The literature on this subject is very extensive, but for a nice review see 42 .
} 
for which the giant graviton is static at $r=\sqrt{1-p / N}$ and, say, $\phi=0$ (we can always shift $\phi$ by a constant). The metric becomes,

$$
d s^{2}=-\left(1-r^{2}\right) d t^{2}+2 r^{2} d t d \phi+\frac{1}{1-r^{2}} d r^{2}+r^{2} d \phi^{2}+\left(1-r^{2}\right) d \varphi^{2}
$$

The connection between these coordinates and the plane wave coordinates is shown in appendix $\mathrm{A}$.

The momentum in $\varphi$ is conserved and is given by,

$$
L=\sqrt{\lambda_{Y M}} \int_{0}^{\pi} \frac{d \sigma}{2 \pi} p_{\varphi} \equiv \sqrt{\lambda_{Y M}} \mathcal{J} .
$$

We choose a gauge that distributes the angular momentum $p_{\varphi}$ homogeneously along the string. Furthermore, we choose $\tau$ to coincide with the global time in the metric. Thus, our gauge is

$$
t=\tau, \quad p_{\varphi}=2 \mathcal{J}=\text { const. }
$$

These type of gauges were introduced in 33

We then implement the constraints that follow from varying $A$ and $B$ in (2.37) directly in the action. This allows us to write the Lagrangian in terms of the momenta $p_{r}$ and $p_{\phi}$ and the fields $r$ and $\phi$ and their derivatives. These are the two fields subject to Dirichlet boundary conditions. We get (up to a total derivative in $\tau$ ),

$$
\mathcal{L}=(1-\dot{\phi}) p_{\phi}+\dot{r} p_{r}-\sqrt{a p_{\phi}^{2}+b p_{r}^{2}+2 c p_{r} p_{\phi}+d},
$$

where,

$$
\begin{aligned}
& a=1+\left(1-r^{2}\right)\left(\frac{\phi^{\prime 2}}{4 \mathcal{J}^{2}}+\frac{1}{r^{2}}\right) \\
& b=\left(1-r^{2}\right)\left(\frac{r^{\prime 2}}{4 \mathcal{J}^{2}}+1\right) \\
& c=-\frac{r^{\prime} \phi^{\prime}\left(1-r^{2}\right)}{4 \mathcal{J}^{2}} \\
& d=\frac{4 \mathcal{J}^{2}}{1-r^{2}}+r^{2} \phi^{\prime 2}+\frac{r^{\prime 2}}{1-r^{2}}
\end{aligned}
$$

As usual, dots and primes denote derivatives with respect to $\tau$ and $\sigma$ respectively. The remaining gauge freedom can be fixed by demanding that the equations of motion for $p_{r}, p_{\phi}, r$ and $\phi$ that follow from (2.45) agree with the ones derived from the original action (2.37) [34. This will fix the value of $B$ in terms of $p_{r}, p_{\phi}, r$ and $\phi$. 
Since the momenta $p_{r}$ and $p_{\phi}$ enter the Lagrangian (2.45) algebraically, we can solve for them using their equations of motion and write the action in terms of the fields $r$ and $\phi$ and their derivatives. We get,

$$
\mathcal{L}=-\left(\frac{d}{1-a \tilde{p}_{\phi}^{2}-b \tilde{p}_{r}^{2}+2 c \tilde{p}_{\phi} \tilde{p}_{r}}\right)^{1 / 2}\left[1+(1-\dot{\phi}) \tilde{p}_{\phi}-\dot{r} \tilde{p}_{r}\right]
$$

where,

$$
\begin{aligned}
& \tilde{p}_{\phi}=\frac{\dot{r} c-(1-\dot{\phi}) b}{a b-c^{2}}, \\
& \tilde{p}_{r}=\frac{\dot{r} a-(1-\dot{\phi}) c}{a b-c^{2}} .
\end{aligned}
$$

Finally, we take the limit $\mathcal{J} \rightarrow \infty$ and assume that the time derivatives are of the order $\partial_{0} x^{\mu} \sim 1 / \mathcal{J}^{2}$. This last condition can be made more precise by solving for the time derivatives in terms of the spatial derivatives as in 34. However, in our case this is not necessary because we just want the lowest order in $1 / \mathcal{J}$. Thus, rescaling $\sigma \rightarrow \pi \sigma$, we get to lowest order,

$$
S \approx-L \int d t \int_{0}^{1} d \sigma\left[\frac{r^{2} \dot{\phi}}{1-r^{2}}+\frac{\lambda}{L^{2}}\left(r^{\prime 2}+r^{2} \phi^{\prime 2}\right)+\mathcal{O}\left(\frac{\lambda^{2}}{L^{4}}\right)\right] .
$$

We note that $L$ serves as an inverse "Planck constant" and so $L \rightarrow \infty$ corresponds to a classical limit as promised. This coordinates are subject to Dirichlet boundary condition, which are expressed as,

$$
\begin{aligned}
& \left.r\right|_{\sigma=0,1}=\sqrt{1-\frac{p}{N}}, \\
& \left.\phi\right|_{\sigma=0,1}=\text { const. }
\end{aligned}
$$

\section{Open Strings on Giant Gravitons from $\mathcal{N}=4 \mathrm{SYM}$}

In this section we study the dual description of open strings on giant gravitons given by $\mathcal{N}=4 \mathrm{SYM}$. It is very interesting to see how the complete geometrical picture discussed in the last section is recovered from the gauge theory. In particular, we show the emergence of the BMN spectrum, the Polyakov action, the Dirichlet boundary conditions and the reparametrization invariance of the string world sheet. All of these geometrical ingredients are encoded in the matrix of anomalous dimension of the operators dual to giant gravitons with open string excitations.

There is considerable evidence that the dual operators to these D3-branes are of determinant-like form [18, 19, 23] (see appendix Q for our conventions)

$$
\mathcal{O}^{p}=\epsilon_{i_{1} \cdots i_{p}}^{j_{1} \cdots j_{p}} Z_{j_{1}}^{i_{1}} \cdots Z_{j_{p}}^{i_{p}}
$$


where $Z$ is one of the three complex scalars of $\mathcal{N}=4 \mathrm{SYM}$. These are chiral operators and, by supersymmetry, their dimensions are determined in terms of their R-charge: $\Delta=J$ (BPS condition). For $\mathcal{O}^{p}$ we see that $\Delta=p \leq N$, and hence this operator obeys the "momentum" bound of the giant gravitons. This makes sense from the AdS/CFT correspondence since we should identify the $U(1)$ charge of (3.1) as the angular momentum of the D-brane and $\Delta$ as its energy.

The operator dual to a single string attached to an $S^{5}$ giant graviton of momentum $p$ is obtained by appending a word to the determinant-like operator [22, 24], which represents the open string ${ }^{3}$,

$$
\mathcal{O}_{W}^{p}=\epsilon_{i_{1} \cdots i_{p}}^{j_{1} \cdots j_{p}} Z_{j_{1}}^{i_{1}} \cdots Z_{j_{p-1}}^{i_{p-1}} W_{j_{p}}^{i_{p}},
$$

where $W$ is a "word" built out of the scalar fields $X, \bar{X}, Y, \bar{Y}, Z, \bar{Z}$, as long as we only consider excitations on the $S^{5}$ directions. Each scalar field carries a unit of $U(1)$ charge corresponding to a unit of angular momentum in one of the three orthogonal planes that cut $S^{5}$ in the dual string picture.

We also have the condition that the $Z$ field is not allowed to be at the borders of $W$ [24]. This constraint comes from the fact that when a $Z$ is at the border of $W$, the operator factorizes as a bigger giant plus a giant of the same size with a closed string (see appendix Q). In fact, in general, the operator (3.2) can be expanded in terms of traces [23]. However, for $p$ sufficiently large the mixing of (3.2) with closed strings (traces) is suppressed in the large $N$ limit. For this reason we will only consider operators with $\sqrt{N} \lesssim p \leq N$.

\subsection{Open Strings as Variable Length Spin Chains}

We are interested in computing the mixing matrix of anomalous dimension for this type of operators at the one-loop approximation and large $N$ limit. Thus, we will only consider planar diagrams. As usual, we begin by defining the correlation function

$$
M_{A B}=\left\langle\tilde{\mathcal{O}}_{A}^{*}(x) \tilde{\mathcal{O}}_{B}(0)\right\rangle_{\text {free }+ \text { interacting }},
$$

where the operators have been normalized according to

$$
\tilde{\mathcal{O}}_{A}(x)=\frac{\mathcal{O}_{A}(x)}{\left\langle\mathcal{O}_{A}^{*}(x) \mathcal{O}_{A}(0)\right\rangle_{\text {free }}^{1 / 2}},
$$

and where $A, B$ are collective indices labeling different giant graviton configurations with a single string. At one-loop and in the large $N$ limit, $M_{A B}$ will have the following general form,

$$
M_{A B}=\frac{1}{|x|^{2 \Delta_{0}}}\left(\delta_{A B}-2 \Gamma_{A B} \log (|x| \Lambda)+\ldots\right),
$$

\footnotetext{
${ }^{3}$ More precisely, as we will see later, the dual operator to the classical non-maximal giant graviton is actually a coherent state of operators like (3.2) with different values of $p$.
} 
where $\Delta_{0}$ is the classical dimension, $\Gamma_{A B}$ is the matrix of anomalous dimension and $\Lambda$ is an ultraviolet cutoff. We then identify the anomalous dimension matrix with the Hamiltonian of the corresponding string quantum states,

$$
\Gamma_{A B} \cong\left\langle\psi_{A}|H| \psi_{B}\right\rangle,
$$

where $\tilde{\mathcal{O}}_{A} \cong\left|\psi_{A}\right\rangle$. This is just the operator state correspondence which is available for any CFT: the Hamiltonian corresponds to taking radial time and compactifying the CFT on a round sphere.

In 25] the case of a maximal giant graviton was considered and it was shown that the resulting planar anomalous dimension matrix corresponds to an integrable open spin chain with $S O(6)$ symmetry. For the case of non-maximal giants, most of the field contractions are the same as with the maximal giants, the difference being the particular combinatorics of the $\epsilon$ symbol for $p \neq N$. However as we remarked in [26], there is a very important new interaction in the case of the non-maximal giant graviton: $Z$ fields can be exchanged between the word $W$ and the rest of the operator. This accounts for the exchange of angular momentum between the giant and the string since the open string is "dragged" by the non-trivial movement of the non-maximal giant graviton.

The details of the combinatorics involved for this and the rest of the interactions between the fields in the operator are explained in the appendix C. Here we just quote the main result focusing on a $S U(2)$ subsector involving the fields (say) $Y$ and $Z^{4}$. There are two kind of terms in the mixing matrix of anomalous dimension. Those corresponding to bulk interactions in the spin chain Hamiltonian, which where already present in the maximal case. They are nothing but the bulk terms of a $S U(2) \mathrm{XXX}$ spin chain. The non-maximality of the giant gives rise to new terms proportional to the quantity $\alpha \equiv \sqrt{1-p / N}$. The most important is a boundary term for the spin chain Hamiltonian, coming from the correlation function representing the exchange of a $Z$ field between the word $W$ and the determinant

$$
\left\langle\tilde{\mathcal{O}}_{W}^{p+1 *}(x) \tilde{\mathcal{O}}_{W^{\prime}}^{p}(0)\right\rangle \sim-\frac{2 \lambda \log (\Lambda|x|)}{|x|^{2 \Delta_{0}}} \sqrt{1-\frac{p}{N}}, \quad \text { with, } \quad \begin{aligned}
& W=Y w \\
& W^{\prime}=Y Z w .
\end{aligned}
$$

This correlation function introduces a variability in the spin chain length at the boundaries. However, it is not a priori clear how to deal with this $S U(2)$ spin chain of variable length. In the following we present how, by relabeling the operators, we can translate the spin chain of variable length into a more manageable bosonic lattice of fixed length and with sources/sinks of bosons at the boundaries.

The most general word in this sector is completely specified by the number of $Z$ fields between two consecutive $Y$ fields,

$$
\left(Y Z^{n_{1}} Y Z^{n_{2}} Y \ldots Y Z^{n_{L}} Y\right)_{i}^{j} \cong\left|n_{1}, n_{2}, \ldots, n_{L}\right\rangle .
$$

\footnotetext{
${ }^{4}$ The labeling of the scalar fields by $Y$ and $Z$ is done on purpose to make the connection with the coordinates used in section 2.3 more explicit.
} 
Thus, the word has a fixed number $L+1$ of $Y$ fields, representing $L+1$ units of angular momentum in $Y$ direction in the string. The number of $Z$ fields and the corresponding angular momentum is measured by the number operator $\hat{n}=\sum_{i} \hat{n}_{i}$. The size of the giant graviton is measured by $\hat{p}=(p+1) \hat{I}-\hat{n}$, where $p$ is the (fixed) total number of $Z$ fields in the operator. The exchange of $Z$ fields occurs only at the first and last site of the bosonic lattice. We will see that the choice of leaving fixed the number of $Y$ fields in our labeling is in correspondence with the gauge choice (2.44) in the Polyakov action.

Since there is equal probability of a $Z$ entering or leaving the word (see appendix C), one expects that for sufficiently large $p$ the lowest energy states have $(\langle\hat{p}\rangle-p) / N \sim$ 0 in the large $N$ limit. That is, the backreaction to the size of the giant graviton should be negligible. Therefore, in what follows we assume that $p \sim \gamma N$ with $0<\gamma \leq 1$, and approximate $\hat{p} / N \approx p / N$ in all matrix elements of the Hamiltonian. Later, we will see that this is a consistent approximation.

The Hamiltonian dual to the anomalous dimension matrix for this sector then takes the form

$$
\begin{aligned}
H= & 2 \lambda \sum_{l=1}^{L} \hat{a}_{l}^{\dagger} \hat{a}_{l}-\lambda \sum_{l=1}^{L-1}\left(\hat{a}_{l}^{\dagger} \hat{a}_{l+1}+\hat{a}_{l} \hat{a}_{l+1}^{\dagger}\right) \\
& +2 \lambda \alpha^{2}+\lambda \alpha\left(\hat{a}_{1}^{\dagger}+\hat{a}_{1}\right)+\lambda \alpha\left(\hat{a}_{L}^{\dagger}+\hat{a}_{L}\right),
\end{aligned}
$$

where $L \lesssim \sqrt{N}$ and the operators $\hat{a}_{i}$ obey the Cuntz algebra for a single species [43],

$$
\hat{a}_{i} \hat{a}_{i}^{\dagger}=I, \quad \hat{a}_{i}^{\dagger} \hat{a}_{i}=I-|0\rangle\langle 0|
$$

where operators corresponding to different sites commute.

For our purposes it will be more useful to think of this algebra as the $q \rightarrow 0$ limit of the deformed Weyl algebra, $\hat{a} \hat{a}^{\dagger}-q \hat{a}^{\dagger} \hat{a}=1,\left[\hat{n}, \hat{a}^{\dagger}\right]=\hat{a}^{\dagger},[\hat{n}, \hat{a}]=-\hat{a}$. The number operator $\hat{n}$ can be constructed in terms of the oscillator operators as in 444.

Terms in the first line of the Hamiltonian (3.9) come from the bulk interactions that were already present in the maximal case. The first term indicates that each bosonic oscillator contributes with an energy $2 \lambda$ whenever its site is occupied. The second term is a hopping interaction for bosons to move between sites, so that the energy is reduced with bosons which are not localized. The second line of (3.9), apart from the constant term, provides source and sink terms at the boundaries of the bosonic lattice, which give rise to non-diagonal boundary conditions, since the total boson occupation number does not commute with the Hamiltonian.

In the next subsections we will discuss two different sectors of the Hamiltonian (3.9) and how they give rise to the BMN limit of short strings and the semiclassical limit of long strings discussed above. 


\subsection{Evidence for a BMN Limit}

At this point, it could be a significant verification of the validity of our dual description to see the BMN spectrum (2.35) arising from the Hamiltonian (3.9). Unfortunately, we have not been able to diagonalize the Hamiltonian (3.9). Nevertheless, evidence for its integrability will be given in section 5 .

However, we do know the ground state:

$$
\left|\Psi_{0}\right\rangle=\left(1-\alpha^{2}\right)^{L / 2} \sum_{n_{1}, \ldots, n_{L}=0}^{\infty}(-\alpha)^{n_{1}+\cdots+n_{L}}\left|n_{1}, \ldots, n_{L}\right\rangle,
$$

and it has energy $E=0$. The expectation value of the number operator for the ground state is,

$$
\left\langle\Psi_{0}|\hat{n}| \Psi_{0}\right\rangle=\frac{L N}{p}\left(1-\frac{p}{N}\right)
$$

which is generically of order $L$, unless $p<<N$. Since $L \lesssim \sqrt{N}$ we see that the backreaction to the giant is indeed small compared to $p \sim N$. Moreover, note that setting $\alpha=0$ gives the familiar ferromagnetic ground state of the maximal giant graviton:

$$
\lim _{\alpha \rightarrow 0}\left|\Psi_{0}\right\rangle=|0,0, \ldots, 0\rangle \simeq(Y Y \cdots Y)_{i}^{j} .
$$

The expectation value (3.12) gives the amount of angular momentum that the string acquires in the direction of the movement of giant. Dividing by the fixed number of $Y$ fields we obtain the ratio of angular momentum in the two directions of the $S^{5}$.

$$
\frac{J_{\psi}}{J_{\eta}} \cong \frac{\text { number of } Z \mathrm{~s} \text { in } W}{\text { number of } Y \mathrm{~S} \text { in } W} \approx \frac{\left\langle\Psi_{0}|\hat{n}| \Psi_{0}\right\rangle}{L}=\frac{N}{p}\left(1-\frac{p}{N}\right) .
$$

This is precisely the same ratio of angular momentum components of the null-geodesic we used to take the Penrose limit (2.10). Thus, as expected, the ground state (3.11) corresponds to a point like string traveling with the giant, and small fluctuations around it should correspond to the modes of the open string in the pp-wave background. Unfortunately we cannot solve for the lowest energy modes of (3.9) in gen-

eral. However, we can treat perturbatively the boundary terms $(\alpha=\sqrt{1-p / N} \leq 1)$ and try to reproduce the spectrum (2.35) at lowest order in $\alpha$.

Before doing this, a few comments are in order. First, let us look at the case of the maximal giant graviton $(\alpha=0)$. In this case there is a precise dictionary between the plane wave excitations and the corresponding gauge theory operators [22]. The ground state (3.13) is excited by adding $Z$ fields to the word $W$ with some 
momentum determined by the boundary conditions. For example, the first excitation is given by a single $Z$ :

$$
\left|\psi_{n}^{1}\right\rangle=\sqrt{\frac{2}{L+1}} \sum_{l=1}^{L} \sin \left(\frac{n \pi l}{L+1}\right)|l\rangle, \text { where }|l\rangle \cong\left(Y^{l} Z Y^{L+1-l}\right)_{i}^{j} .
$$

The anomalous dimension of this operator is,

$$
E_{n}^{(0)}=2 \lambda\left[1-\cos \left(\frac{n \pi}{L+1}\right)\right] \approx \frac{\lambda \pi^{2} n^{2}}{L^{2}},
$$

On the other hand, the first excited state of the open string in the pp-wave background has energy,

$$
E_{n(\text { String })} \approx 1+\frac{\lambda \pi^{2} n^{2}}{L^{2}}
$$

The factor of 1 can be interpreted from the increase of the classical dimension of the word by the insertion of a single $Z$ field. Then, the anomalous dimension (3.16) agrees with the small correction in (3.17).

However, for the case of the non-maximal giant graviton there is no precise notion of the length of the word, and hence its classical dimension. This is because $Z$ letters can enter and leave the word. This means that we do not have a clear cut distinction between what we call the "string" and the "giant". In fact, we only know the average length of the word to leading order in $L: L+1+\langle n\rangle \sim \mathcal{O}(L)$. Adding or subtracting a finite number of $Z$ fields to our operators will have no effect in the anomalous dimension in the planar approximation.

In the spectrum of open strings on the pp-wave geometry we found what appears to be two independent energy modes (2.35) differing by a factor of even integers. But this is precisely the ambiguity in the classical dimension of the corresponding operators. Therefore, we should only compare the anomalous dimensions of our operators to the $\mathcal{O}\left(\lambda / L^{2}\right)$ corrections in the string theory spectrum. In fact, we see that setting $\alpha=0$ in (2.35) gives precisely the anomalous dimension (3.16).

We now want to calculate the lowest order correction in $\alpha$ to the energy of the lowest BMN excitations of the maximal giant. For the sake of simplicity, we focus on the eigenstates with one-boson; that is, a single $Z$ on the word. We write the Hamiltonian as

$$
H=2 \lambda \alpha^{2}+H_{0}+\alpha V
$$

where

$$
H_{0}=2 \lambda \sum_{l=1}^{L} \hat{a}_{l}^{\dagger} \hat{a}_{l}-\lambda \sum_{l=1}^{L-1}\left(\hat{a}_{l}^{\dagger} \hat{a}_{l+1}+\hat{a}_{l} \hat{a}_{l+1}^{\dagger}\right)
$$

and

$$
V=\lambda\left(\hat{a}_{1}^{\dagger}+\hat{a}_{1}+\hat{a}_{L}^{\dagger}+\hat{a}_{L}\right)
$$


The ground state $\left|\psi^{0}\right\rangle$ of the unperturbed Hamiltonian $H_{0}$ has zero occupation number and zero energy (c.f. (3.13)). The one-boson eigenstates and eigenenergies were given in Eqs. (3.15) and (3.16).

The corrected energy is

$$
E_{n}=2 \lambda \alpha^{2}+E_{n}^{(0)}+\alpha V_{n n}+\alpha^{2} \sum_{k \neq n} \frac{\left|V_{n k}\right|^{2}}{E_{n}^{(0)}-E_{k}^{(0)}}+\mathcal{O}\left(\alpha^{3}\right) .
$$

Matrix elements of $V$ in the unperturbed basis are non-vanishing only for eigenstates that differ in one boson. Then, $V_{n n}$ is zero and the first correction is order $\alpha^{2}$. To compute the correction to the one-boson eigenvalue at this perturbative order, the only matrix elements needed are

$$
\left\langle\psi_{n}^{1}|V| \psi^{0}\right\rangle=\lambda \sqrt{\frac{2}{L+1}} \sin \left(\frac{n \pi}{L+1}\right)\left(1-(-1)^{n}\right), \quad \text { and } \quad\left\langle\psi_{n}^{1}|V| \psi_{m}^{2}\right\rangle,
$$

where $\left|\psi_{m}^{2}\right\rangle$ are the unperturbed two-boson eigenstates (in this case $m$ is a collective index). However, it is hard to write down the two-boson eigenstates in closed form. One can not use a dilute gas approximation, because this would only be valid for small energies and we need $\left|\psi_{m}^{2}\right\rangle$ for all energies. Still, it is possible diagonalize numerically $H_{0}$ in the two-boson subspace, i.e. to solve the eigenvalue problem for a $L(L+1) / 2 \times L(L+1) / 2$ matrix, for different finite values of $L$ and use the results to compute the energy correction. Then, we can see that as the number of sites increases, the correction not only captures the $1 / L^{2}$ dependence but also the proportionality factor tends to the one predicted by the plane-wave spectrum

$$
E_{n} \simeq \lambda \frac{\pi^{2} n^{2}}{L^{2}}\left(1-2 \alpha^{2}\right) .
$$

For instance, in table 1 we show the shift of the $n=1$ one-boson eigenvalue divided by the predicted shift (3.23).

Table 1: Shift in $n=1$ one-boson energy.

\begin{tabular}{|c|c|}
\hline$L$ & $-\frac{\Delta E_{1}}{2 \pi^{2} /(L+1)^{2}}$ \\
\hline 15 & 1.122 \\
20 & 1.091 \\
25 & 1.079 \\
30 & 1.060 \\
\hline
\end{tabular}

If our Hamiltonian is integrable (see section 5), then maybe some generalization of the Bethe Ansatz can be used to diagonalize it and provide the final proof of 
the presence of the BMN limit. Integrability is not strictly necessary. There are other forms of obtaining the BMN limit exactly at strong coupling using different techniques 45, 31, 46], see also [47. We also have uncovered some other qualitative features of the spectrum including the presence of continuum bands by doing perturbative diagrams that cast doubt on a solutions of the problem in terms of a Bethe Ansatz. We discuss these in section 4 .

\subsection{The Semiclassical Limit}

In general, it is possible to obtain a semiclassical sigma model action governing the dynamics of a given spin chain. In 32 it was shown that the sigma model obtained from the spin chain associated to the $S U(2)$ sector of SYM coincides with the Polyakov action describing the propagation of closed strings in $A d S_{5} \times S^{5}$, in a particular limit. This comparison was extended to other sectors 48, 49] and to other realizations of the AdS/CFT correspondence [50, 51, 52].

Now we will obtain a semiclassical sigma model action that governs the dynamics of bosonic lattice in the $L \rightarrow \infty$ limit, using the coherent states basis for the path integral representation of the evolution operator. This was already discussed in our previous note [26]. For completeness, we will use the $q$-deformed Weyl algebra and will set $q \rightarrow 0$ when needed. In appendix $\mathbb{E}$, we give the definition of the coherent states for this algebra.

One can then put a coherent state at each site of the bosonic lattice and construct the propagator between the coherent states in the usual way (see [53]). The resulting action is,

$$
\begin{aligned}
S= & \int d t\left(i\left\langle z_{1} \ldots z_{L}\left|\frac{d}{d t}\right| z_{1} \ldots z_{L}\right\rangle-\left\langle z_{1} \ldots z_{L}|H| z_{1} \ldots z_{L}\right\rangle\right) \\
= & \int d t\left[-\sum_{i=1}^{L} f_{q}\left(r_{i}\right) \dot{\phi}_{i}-2 \lambda\left(\alpha^{2}+\alpha\left(r_{1} \cos \phi_{1}+r_{L} \cos \phi_{L}\right)\right)\right. \\
& \left.\left.-2 \lambda\left(\sum_{i=1}^{L} r_{i}^{2}-\sum_{i=1}^{L} r_{i} r_{i+1} \cos \left(\phi_{i}-\phi_{i+1}\right)\right)\right)\right],
\end{aligned}
$$

where we defined,

$$
f_{q}(x)=x^{2} \frac{\exp _{q}^{\prime}\left(x^{2}\right)}{\exp _{q}\left(x^{2}\right)}
$$

In the large $L$ limit, the complex modulus $r$ and the complex argument $\phi$ become functions of a continuous variable $0 \leq \sigma \leq 1$. We consider the action (3.24) in the limit $L \rightarrow \infty$ and $\lambda \rightarrow \infty$ but keeping $\lambda / L^{2}$ fixed and small. The result is the 
following sigma model,

$$
\begin{aligned}
S= & -L \int d t \int_{0}^{1} d \sigma\left[f_{q}(r) \dot{\phi}+\frac{\lambda}{L^{2}}\left(r^{\prime 2}+r^{2} \phi^{\prime 2}\right)\right] \\
& -\left.\lambda \int d t\left[\alpha^{2} \sin ^{2} \phi+(\alpha \cos \phi+r)^{2}\right]\right|_{\sigma=0} \\
& -\left.\lambda \int d t\left[\alpha^{2} \sin ^{2} \phi+(\alpha \cos \phi+r)^{2}\right]\right|_{\sigma=1},
\end{aligned}
$$

where dots and primes refer to derivatives with respect to $t$ and $\sigma$ respectively. If we take the limit $q \rightarrow 0$, the function above reduces to $f_{q}(r) \rightarrow r^{2} /\left(1-r^{2}\right)$ and we see that the bulk action of (3.24) coincides with the Polyakov action in the large momentum limit (2.53). This gives a direct geometrical meaning to the fields $r$ and $\phi$ of the coherent states: as spacetime coordinates.

The classical Hamiltonian of the coherent states is

$$
\begin{aligned}
\langle H\rangle & =\frac{\lambda}{L} \int_{0}^{1} d \sigma\left(r^{\prime 2}+r^{2} \phi^{\prime 2}\right)+\left.\lambda\left[\alpha^{2} \sin ^{2} \phi+(\alpha \cos \phi+r)^{2}\right]\right|_{\sigma=0} \\
& +\left.\lambda\left[\alpha^{2} \sin ^{2} \phi+(\alpha \cos \phi+r)^{2}\right]\right|_{\sigma=1} .
\end{aligned}
$$

One sees that the boundary terms will give rise to a large anomalous dimension of order $\sim \lambda$ unless,

$$
\begin{aligned}
& \left.r\right|_{\sigma=0,1}=\alpha=\sqrt{1-\frac{p}{N}}, \\
& \left.\phi\right|_{\sigma=0,1}=\pi .
\end{aligned}
$$

These are exactly the Dirichlet boundary conditions 2.54) on open strings on nonmaximal giant gravitons! The fact that the boundary terms give rise to a large anomalous dimension is nothing but the statement that moving the D-brane takes a lot of energy.

In the limit $L \rightarrow \infty$ we get the following classical equations of motion:

$$
\begin{aligned}
\frac{r \dot{r}}{\left(1-r^{2}\right)^{2}}+\frac{\lambda}{L^{2}} \partial_{\sigma}\left(r^{2} \phi^{\prime}\right) & =0, \\
\frac{r \dot{\phi}}{\left(1-r^{2}\right)^{2}}+\frac{\lambda}{L^{2}}\left(r \phi^{\prime 2}-r^{\prime \prime}\right) & =0 .
\end{aligned}
$$

Looking at the Hamiltonian (3.27) we see that the classical solutions to these equations will have energies $\sim \lambda / L$, which represent a small multiplicative correction to their "bare" energies: $L+1+\langle n\rangle+\mathcal{O}(\lambda / L) \sim L\left(\right.$ const. $\left.+\mathcal{O}\left(\lambda / L^{2}\right)\right)$. This is already a familiar result for closed semiclassical strings 42 .

The average number of bosons in the lattice is,

$$
\langle\hat{n}\rangle=L \int_{0}^{1} d \sigma \frac{r^{2}}{1-r^{2}} .
$$


This provides a way of measuring length of the spin chain in the original XXX model formulation. In general, $\langle\hat{n}\rangle$ is not conserved and therefore the string will oscillate in length. Using Eq. (3.30), this variation can be put simply as,

$$
\partial_{t}\langle\hat{n}\rangle=2 \frac{\lambda}{L}\left(1-\frac{p}{N}\right)\left(\left.\phi^{\prime}\right|_{\sigma=0}-\left.\phi^{\prime}\right|_{\sigma=1}\right) .
$$

Note however that we must ensure that $\langle\hat{n}\rangle$ is bounded so that neglecting the backreaction to the giant remains a good approximation.

Moreover, we see how the particular choice of spacetime coordinates and world sheet gauge are encoded in the SYM side. We labeled our states using the word $W$ only, which is dual to the open string. This is translated to the string side by choosing a coordinate system for which the giant graviton is static. Finally, by labeling the states as in (3.8) we are explicitly distributing the angular momentum in $Y$ uniformly along the string. This has very strong implications in the AdS/CFT correspondence, because we are seeing explicitly the reparametrization invariance of the string worldsheet: the gauge that makes the calculation more natural is different than the one considered in other semiclassical setups [39, 34].

\section{A D-brane Instability?}

He have seen that the anomalous dimensions of the dual operators of non-maximal giant gravitons with open strings excitations is described in terms of a spin chain of variable length. We offered an alternative description in terms of a bosonic lattice. There, the variability of the spin chain's length was translated into the variability of the boson occupation number. In this section, we study the possibility of having configurations with the occupation number growing monotonically in time. More precisely, we show evidence for the presence of continuum energy bands in the spectrum of the Hamiltonian (3.9). If a configuration has enough energy to access the continuum, a more general time dependence than an oscillation is allowed for its mean occupation number.

In particular, we argue that in the large $L$ limit there is at least one band that is accessible to classical long strings with energies $\sim \lambda / L$. This has profound consequences for the stability of the D-branes because the word $W$ in (3.2) can be excited in such a way to absorb a large number of $Z$ fields from the giant graviton. In the dual picture, this means that the string becomes longer and longer absorbing more and more angular momentum from the D-brane. A long string attached to the moving giant graviton, could suffer centrifugal forces, consequence of a non-geodesic movement, and if the string is long enough its tension could be overwhelmed by

these forces. Finally, the D-brane would become small enough so that the mixing amplitude with closed strings is no longer negligible (see appendix Q). In fact, way before this happens, the string would have absorbed $\mathcal{O}(N) Z$ fields so that the 
planar approximation used in the calculations will be invalidated. The outcome of the instability is then difficult to predict but can have important consequences in string theory.

\subsection{One Site}

We start with the Hamiltonian for $L=1$ :

$$
H=2 \lambda\left[\alpha^{2}+\hat{a}^{\dagger} \hat{a}+\alpha\left(\hat{a}^{\dagger}+\hat{a}\right)\right] .
$$

The advantage of this case is that we can diagonalize $H$ explicitly for $q=0$. This is because $\hat{a}^{\dagger}$ and $\hat{a}$ act as shift-left and shift right operators for $q=0$. Thus, the matrix representation of $H$ looks like the following matrix

$$
H \sim \alpha^{2}+\left(\begin{array}{ccccc}
0 & \alpha & 0 & 0 & \ldots \\
\alpha & 1 & \alpha & 0 & \ldots \\
0 & \alpha & 1 & \alpha & \ddots \\
\vdots & \ddots & \ddots & \ddots & \ddots
\end{array}\right),
$$

and this is the same hamiltonian for a particle hopping on a semi-infinite lattice, as well as the quadratic form that one considers when studying the potential energy of a semi-infinite collection of beads attached by springs.

The complete set of eigenstates consists of the ground state (3.11) with $L=$ 1 , which is considered as a bound state for the hopping particle to scatter of the boundary and the continuum,

$$
|\Psi(k)\rangle=\sum_{n=0}^{\infty}[\sin k n+\alpha \sin k(n+1)]|n\rangle, \quad \text { with } 0 \leq k \leq \pi,
$$

and energy

$$
E(k)=2 \lambda\left(1+2 \alpha \cos k+\alpha^{2}\right) .
$$

The gap with the ground state turns out to be

$$
2 \lambda(1-\alpha)^{2} .
$$

Moreover, the maximal energy of the continuum is $2 \lambda(1+\alpha)^{2}$. These continuum states follow a delta-function normalization

$$
\left\langle\Psi\left(k^{\prime}\right) \mid \Psi(k)\right\rangle=\frac{\pi}{2}\left(1+2 \alpha \cos k+\alpha^{2}\right) \delta\left(k^{\prime}-k\right) .
$$

We can then use them to build normalizable wave-packets

$$
|\phi\rangle=\int_{0}^{\pi} d k f(k)|\Psi(k)\rangle,
$$


choosing a function $f(k)$ so that $\langle\phi \mid \phi\rangle=1$ and the initial occupation number $\langle\phi|\hat{n}(0)| \phi\rangle$ is finite. Then we can have normalized states with finite energy but with growing occupation number.

For instance, the wave-packet

$$
|\phi\rangle \propto \int_{0}^{\pi} d k \sin k|\Psi(k)\rangle=\frac{\pi}{2}(\alpha|0\rangle+|1\rangle),
$$

increases its mean occupation number while it evolves, as it is depicted in Figure 1.

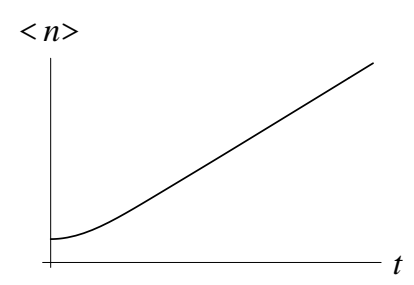

Figure 1: Mean occupation of wave-packet (4.8).

This continuum spectrum feature is not only a property of $q=0$. Indeed, when $q<1$ we always find the same behavior. This is because for sufficiently large $n$ (occupation number), the hamiltonian asymptotes exponentially quickly to a similar form

$$
H \sim\left(\begin{array}{cccccc}
\ddots & \ddots & \ddots & \ddots & \ddots & \ddots \\
\ddots & \alpha & 1 & \alpha & \ddots & \ddots \\
\ddots & \ddots & \alpha & 1 & \alpha & \ddots \\
\ddots & \ddots & \ddots & \ddots & \ddots & \ddots
\end{array}\right),
$$

since $q^{n} \rightarrow 0$.

\subsection{Two Sites}

For two sites we can no longer solve for the exact spectrum but we can do (degenerate) perturbation theory around the maximal case $(\alpha=0$ and $q=0)$. The unperturbed Hamiltonian for two sites is,

$$
H_{0}=2 \lambda \hat{a}_{1}^{\dagger} \hat{a}_{1}+2 \lambda \hat{a}_{2}^{\dagger} \hat{a}_{2}-\lambda\left(\hat{a}_{1}^{\dagger} \hat{a}_{2}+\hat{a}_{1} \hat{a}_{2}^{\dagger}\right) .
$$

Since this Hamiltonian correspond to an integrable spin chain, we can diagonalize it using the usual Bethe Ansatz. The eigenstates are (e.g. see [25]),

$$
\left|\Psi_{n}^{(0)}(k)\right\rangle=A_{n}(k) \sum_{l=0}^{n}[2 \sin (k l)-\sin (k(l+1))]|l, n\rangle,
$$


where the states $\left|n_{1}, n_{2}\right\rangle$ have been re-labeled $\left|n_{1}, n_{1}+n_{2}\right\rangle \equiv|l, n\rangle$ to reflect the fact that the number operator commutes with $H_{0}$. The energy of these eigenstates is given by,

$$
E(k)=2 \lambda(2-\cos k),
$$

and the complex momentum $k$ is determined by the equation,

$$
4 \sin (k n)-4 \sin (k(n+1))+\sin (k(n+2))=0 .
$$

We now want to consider first order perturbation theory in $\alpha$ around these eigenstates. Again, the perturbing potential is (3.20) and we ignore the constant term in (3.18) which is $O\left(\alpha^{2}\right)$. In particular we are interested in the continuum energy bands that could arise from turning on the perturbation. For that to occur we will need to have an infinite degeneracy or an accumulation point in $k$ in the large $n$ limit.

Let us start by looking at the infinite degenerate roots of (4.13). It is easy to show that for a given $n$, the degenerate roots have the form $k=a \pi / b$ with $a, b \in \mathbb{Z}$ and where the degeneracy occurs for states $\left|\Psi_{n^{\prime}}^{(0)}\right\rangle$ with $n^{\prime}=n+\mathbb{Z} b$. Now, the perturbation (3.20) has non-zero matrix elements only between unperturbed states differing in $n$ by one. Therefore, at first order in perturbation theory we only need to consider the degenerate roots: $k=0, \pi$ (we can take $k \in[0, \pi]$ without loss of generality).

For $k=0$, the unperturbed energy is $E^{(0)}=2 \lambda$. To find the first order correction in $\alpha$ we need to diagonalize $V$ in the subspace of the unperturbed energy eigenstates (4.11). However for our purposes, we only need to look at the large $n$ limit to see the presence of the continuum. One can show that in this limit,

$$
V\left|\Psi_{n}^{(0)}\right\rangle \sim 2 \lambda \alpha\left(\left|\Psi_{n+1}^{(0)}\right\rangle+\left|\Psi_{n-1}^{(0)}\right\rangle\right) .
$$

Thus the asymptotic form of the first order correction to the eigenstates is going to be of the form of a superposition of plane waves $\left|\Psi_{n}^{(1)}\right\rangle \sim \sum_{n} e^{i p n}\left|\Psi_{n}^{(0)}\right\rangle$ with continuum momentum $p$ and energy:

$$
E^{(1)}=4 \alpha \lambda \cos p .
$$

If we follow the same procedure with the root $k=\pi$ we find that at large $n$ the matrix elements of $V$ vanish and so there is no continuum band at this energy. Next, we consider the case of complex $k$ and we find an exponential accumulation point at $k=i \log 2$ with energy $E^{(0)}=3 \lambda / 2$. The asymptotic form of the action of the perturbation is,

$$
V\left|\Psi_{n}^{(0)}\right\rangle \sim \frac{3}{2} \lambda \alpha\left(\left|\Psi_{n+1}^{(0)}\right\rangle+\left|\Psi_{n-1}^{(0)}\right\rangle\right),
$$

and so we get a continuum band with,

$$
E^{(1)}=3 \alpha \lambda \cos p .
$$




\subsection{Multiple Sites}

For multiple sites a quantum analysis is no longer feasible. Nevertheless the presence of continuum bands can be seen directly from a classical analysis. For any number of sites the classical limit is $\hbar \rightarrow 0$ and thus we can use the coherent state Hamiltonian. For completeness, we will work with $0 \leq q \leq 1$ and then take the limit $q \rightarrow 0$ when needed.

Looking at the action (3.24) we see that our system is subject to the constraints,

$$
\begin{aligned}
p_{r_{i}} & =0, \\
p_{\phi_{i}}+f_{q}\left(r_{i}\right) & =0,
\end{aligned}
$$

and then, the phase space is isomorphic to the configuration space $\left(r_{i}, \phi_{i}\right)$, which is a product of $L$ discs $D_{q}$ of radii $\frac{1}{1-q}$. Using the auxiliary constants $r_{0}=r_{L+1}=\alpha$ and $\phi_{0}=\phi_{L+1}=\pi$ the classical Hamiltonian can be simply posed as

$$
\mathcal{H}_{L}=2 \lambda \sum_{i=0}^{L} r_{i}\left(r_{i}-r_{i+1} \cos \left(\phi_{i}-\phi_{i+1}\right)\right) \text {. }
$$

In particular, let us first consider the one-site classical Hamiltonian

$$
\mathcal{H}_{1}=2 \lambda\left(\alpha^{2}+r^{2}-4 \alpha r \cos \phi\right) .
$$

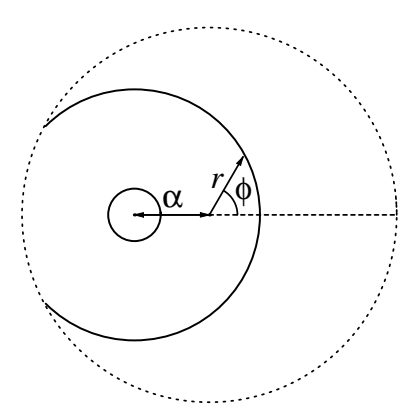

Figure 2: Closed and open orbits in $D_{q}$.

Curves of constant energy, which is the unique conserved quantity, determines the trajectories in the two-dimensional phase space $D_{q}$. In the present coordinates, the $\mathcal{H}_{1}=E$ curves are nothing but circles whose centers are displaced a distance $\alpha$ from the center of the disc $D_{q}$ (see figure Q). The radii of these orbits are given by the energy through $\sqrt{E / 2 \lambda}$. As long as $E<2 \lambda\left(\frac{1}{1-q}-\alpha\right)^{2}$ the orbits are closed circles. However, for energies $2 \lambda\left(\frac{1}{1-q}-\alpha\right)^{2}<E<2 \lambda\left(\frac{2}{1-q}-\alpha\right)^{2}$ the orbits are open arcs. In a semiclassical approach, energies for which the trajectories are open in the phase space give rise to a the continuum in the quantized system. The proper measure of 
the continuum is the phase space area between energies $E$ and $E+\delta E$. This area has to become infinite once the trajectories reach the boundary (otherwise one would find that the phase space has finite area, and then the total number of states would have to be finite).

The minimal and maximal energies of the continuum in the $q \rightarrow 0$ limit are seen to agree with the results of the quantum perturbation theory.

We now finally come to the case of arbitrary number of sites. In this case we will only calculate the energy gap between the ground state and the first continuum band. To obtain the energy for which the continuum begins, one has to look for the minimal energy for which the energy constant hypersurfaces intersect the boundary of the phase space $D_{q}^{L}$. To this end one needs to compute and compare the absolute minimum of the resulting $L$ functions when one of the $r_{i}$ is set to $\frac{1}{1-q}$. These $L$ functions are bounded from below by quadratic polynomials on the radii $r_{i}$

$$
\mathcal{H}_{L}^{j}=\left.\mathcal{H}_{L}\right|_{r_{j}=\frac{1}{1-q}} \geq \mathcal{M}_{L}^{j}=\left.2 \lambda \sum_{i=0}^{L} r_{i}\left(r_{i}-r_{i+1}\right)\right|_{r_{j}=\frac{1}{1-q}}
$$

and coincide with them when all angles $\phi_{i}$ are taken to be $\pi$. Then it suffices to find out the minima of the $\mathcal{M}_{L}^{j}$. The stationary points should satisfy in all cases the recursive equation

$$
2 r_{i}-r_{i+1}-r_{i-1}=0
$$

subject to the boundary conditions

$$
r_{0}=\alpha, \quad r_{j}=\frac{1}{1-q}, \quad r_{L+1}=\alpha .
$$

The recursive equation (4.22) is fulfilled by a linear dependence of $r_{i}$ on the site labeling $i$, with the constants adjusted to also fulfill the boundary conditions (4.23). The critical point of each $\mathcal{M}_{L}^{j}$ is

$$
\begin{array}{ll}
r_{i}^{*}=\left(\frac{1-\alpha(1-q)}{j(1-q)}\right) i+\alpha & \text { if } i \leq j \\
r_{i}^{*}=\left(\frac{\alpha(1-q)-1}{(L+1-j)(1-q)}\right) i+\frac{L+1-j \alpha(1-q)}{(L+1-j)(1-q)} & \text { if } i>j
\end{array}
$$

It is easy to see that each critical point is a minimum of each $\mathcal{M}_{L}^{j}$, since all the eigenvalues of the corresponding Hessians are strictly positive. The energy evaluated in these points is

$$
\left.\mathcal{H}_{L}^{j}\right|_{r_{i}=r_{i}^{*}, \phi_{i}=\pi}=\frac{\lambda(L+1)(1-\alpha(1-q))^{2}}{j(L+1-j)(1-q)^{2}} .
$$

As a function of $j$, that takes values $1 \leq j \leq L$, the minimal energy is obtained for the $j$ corresponding to the central site. So, the minimal energy for which constant 
energy hypersurfaces intersect the phase space boundary is

$$
E_{\text {cont }}=\left\{\begin{array}{cl}
\frac{4 \lambda(1-\alpha(1-q))^{2}}{(L+1)(1-q)^{2}} & \text { if } L \text { is odd } \\
\frac{4 \lambda(L+1)(1-\alpha(1-q))^{2}}{L(L+2)(1-q)^{2}} & \text { if } L \text { is even }
\end{array} .\right.
$$

Let us now turn back to case $q \rightarrow 0$. For $L=1$ we have again $E_{\text {cont }}=2 \lambda(1-\alpha)^{2}$, which is the minimum of the unique band (4.4) of the quantum system. For $L=2$, we have $E_{\text {cont }}=3 \lambda / 2(1-\alpha)^{2}$ which is the minimum of the lowest band (4.17) computed perturbatively for small $\alpha$. We are eventually interested in the limit of a large number of sites $L$. According to the classical analysis, the minimum of the lowest band is, in that case,

$$
E_{\mathrm{cont}}=\frac{4 \lambda(1-\alpha)^{2}}{L}
$$

As we argued, from this energy the quantized system will have a continuum spectrum. Then, this is the amount of energy needed for having configurations with a mean occupation number growing in time. Note that this energy is accessible to long semiclassical strings.

Let us end this section, presenting how the transition from oscillatory to nonoscillatory solutions near the value $E_{\text {cont }}$ is observed in numerical solutions. In figures 3, 4 and 5 we show different classical solutions of the system with $L=30$ and their corresponding occupation numbers. The 3 cases were solved with similar initial data. Only the amplitude of the initial profiles $\left(r_{i}, \phi_{i}\right)$ were slightly varied to change the energies. Moreover we chose the energies to be near the critical value $E_{\text {cont }}$. For energies below $E_{\text {cont }}$, no $r_{i}$ can tend to 1 , so the mean occupation number is kept bounded. But as long as the energy is bigger than $E_{\text {cont }}$ it is possible to find solutions with some $r_{i}$ approaching 1 and a mean occupation number growing monotonically in time.
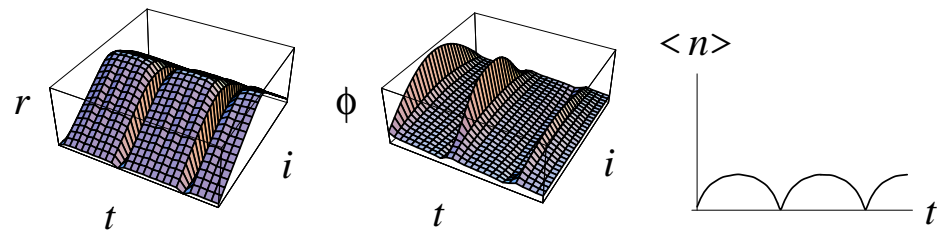

Figure 3: $L=30$ solution with energy $E=0.94 E_{\text {cont }}$.

\subsection{A toy model for the D-brane instability}

We have found, surprisingly, that the spin chain model associated to a string attached to a non-maximal giant exhibits a continuous spectrum. Naively, this is counter to 

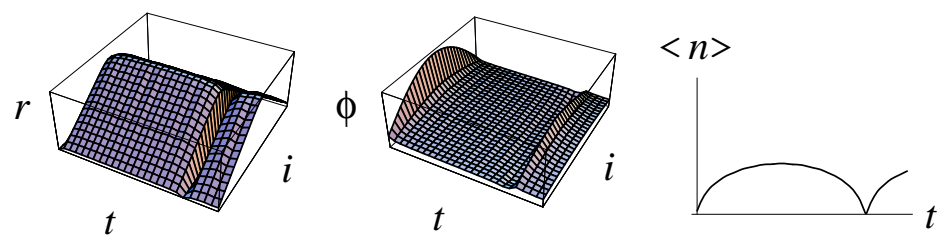

Figure 4: $L=30$ solution with energy $E=0.99 E_{\text {cont }}$.
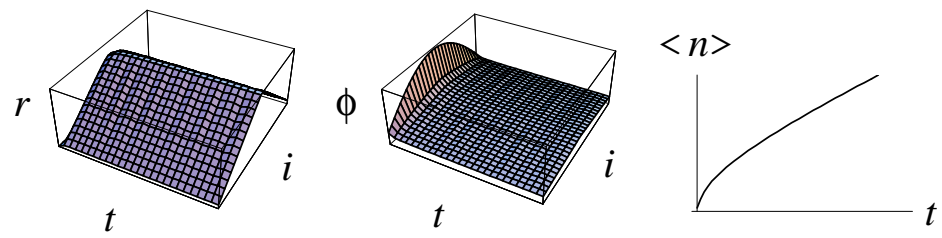

Figure 5: $L=30$ solution with energy $E=1.05 E_{\text {cont }}$.

our intuition from AdS, as we have a finite energy state, and in the dual field theory, there are only finitely many states with energy less than $E$. This means that the true spectrum of the string plus D-brane system ultimately must be discrete.

Considering how we got our spin chain model, we have to notice that we had various assumptions built in our computations. First, the giant has energy of order $N$, while the string should have an energy of order $\sqrt{N}$, roughly, this corresponds to occupation numbers lower than $\sqrt{N}$. This is required for the planar approximation to Feynman diagrams to make sense. If the total angular momentum of the spin chain becomes large with respect to $\sqrt{N}$, the planar approximation breaks down. This means that the Hamiltonian in terms of the boson spin chain we have described is only valid in some regime (where the occupation numbers are not too large), and when we get out of that regime, the fact that $N$ is finite is important. As we take $N$ large, the bosonic spin chain model realm of applicability grows. So the continuum spectrum and bands we have been discussing are a property of the strict large $N$ limit. At finite $N$, the continuum spectrum is resolved into a discrete spectrum with an extremely fine spacing of the eigenvalues. This would be very hard for an observer to determine. This continuum spectrum, which is associated with large quantum numbers for the occupation numbers of the chain has to be interpreted in terms of classical physics (this is the same reasoning that went into the work [54). At large quantum numbers, the problem becomes classical, and we should be able to come up with a classical argument that shows that the strings should grow in size, so long as string interactions and the brane back reaction can be ignored (these are the $1 / N$ effects that the planar approximation does away with).

Now, we want to come up with a model of the system that clearly shows the instability, without the complications of the $A d S_{5} \times S^{5}$ geometry. The idea is simple: 
we have a charged D-brane in the presence of a magnetic RR background. Because of the background, a moving D-brane is accelerated with respect to geodesic free-fall by a Lorentz force. Since fundamental strings are not charged with respect to the RR-background, they should prefer to follow geodesics.

This motivates the following toy model for the giant graviton setup: the ends of the strings are attached to the D-brane, so they are accelerating. By the equivalence principle, we can think of the same system as a string with fixed ends suspended in a gravitational field. A close analogy of the setup is the act of suspending cables between telephone poles. The telephone poles are replaced by the D-brane itself, and it is supported in place by the RR-background. The cable between the telephone posts is the string. Unlike conventional cables, the string tension is constant (a fundamental constant in perturbative string theory). This means that in principle the string can weigh more that the tension of the string can support. This requires a long string (long enough so that the weight of string between the two posts is bigger than the string tension), whose length depends on the strength of the gravitational field.

Since the string can not break (we are assuming that we are in the strict planar approximation), the string will grow by falling, if it is long enough to begin with (this can be accomplished by separating the putative telephone posts sufficiently). In fact, the string will keep on falling forever if string interactions are turned off. Otherwise, the string will get so long that eventually there is a finite probability per unit time that the one long string will break into a closed loop plus a shorter string suspended between the post (this can be understood as the emission of gravitational waves, or other fields, by an accelerated object).

Our toy model clearly exhibits the instability of the string system that we found with our spin chain model. It also provides for the presence of the gap: if the telephone posts are close together, the minimal energy string will be stable. Indeed, many small perturbations of the configuration will be allowed. To make the string fall, we might need to stretch it a lot, but once we have enough weight in the system, the string can not sustain the total weight of the string, and the instability we discuss will have set in. Because we have a regime where the string is stable, this regime of lower energy will have a discrete spectrum, and when we reach the instability, we will get a continuous spectrum. This gap is determined by the distance between the telephone posts (this can be related to the total angular momentum of the string ground state), and by the effective gravitational field. This effective gravitational field is determined by how much is the giant graviton accelerating: the bigger giants move slowly, so their effective gravity is small, while small giants move fast and accelerate a lot more.

This means that the gap in the spectrum should become smaller as the D-brane becomes smaller (in our spin chain notation, $\alpha$ becomes bigger). This is exactly what we see from eq. 4.5, as well as from understanding the details of figure 2 . 


\section{Evidence for Integrability}

In this section we present numerical evidence for the integrability of the classical Hamiltonian (4.19) for $q \in[0,1]$. For $L$ sites, this would correspond to have $L$ constants of motion ${ }^{5}$. Then, trajectories in the $2 L$-dimensional phase space take place in a $L$-dimensional torus. However, with the exception of separable systems, there is no systematic procedure for finding constants of motion. Even in the rather simple case of two sites, we do not know another constant of motion apart from the energy whenever $\alpha \neq 0$. Hypersurfaces of constant energy are 3 -spheres in $D_{q}^{2}$, and if a second constant of motion existed, the motion would be in a 2-torus included in the 3-sphere. Then, the intersection of this 2-torus with a any hypersurface (of dimension 3) would be in general a closed curve in the phase space. We can verify if this is the case by studying numerical solutions of the system. For example, in Figure 6 we plot the values of coordinates $\left(r_{2}, \phi_{1}, \phi_{1}\right)$ of a given solution for many different times when the variable $r_{1}$ takes the value $1 / 2$. The fact that points lie in a closed curve is a strong indication that the motion is taking place in a 2-torus and the system is integrable. One can try this for many different hypersurfaces and one always gets closed curves. Similar evidence is found for cases with higher number of sites.

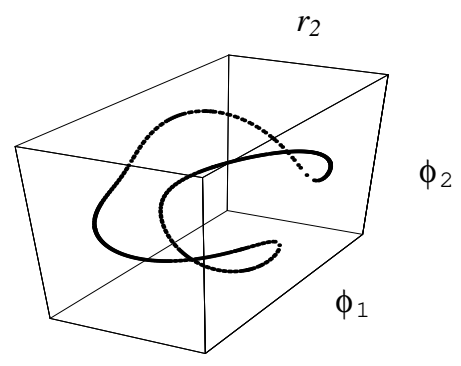

Figure 6: Intersections of the a solution with $r_{1}=1 / 2$.

Note that for $q=1$ the deformed operators (E.1) become the usual harmonic oscillators and the system becomes a lattice of ordinary bosons. In this case quantum and classical integrability follow trivially. For $q \neq 1$ the classical Hamiltonian (4.19) can be seen as a deformation of the Hamiltonians studied in [55, 56, 57]. We now conjecture that our family of Hamiltonians parameterized by $q, \alpha$ is indeed a two parameter family of (quantum) integrable Hamiltonians.

Because the spectrum of the quantum hamiltonian has a continuum, it is very unlikely that the system can be solved via a simple Bethe Ansatz for all $\alpha$, even though for $\alpha=0$ the system can be solved via a Bethe Ansatz. The distinction

\footnotetext{
${ }^{5}$ Moreover the constants of motion should be compatible with each other, which means that the Poisson brackets with each other should vanish.
} 
between $\alpha=0$ and $\alpha \neq 0$ resides in the fact that the constant energy surfaces are compact in the first case, while they are non-compact in the second case (they intersect the boundary). This non-compactness of the energy surfaces for the spin model upsets the usual Bethe Ansatz intuition for finite systems. In finite systems the Bethe ansatz hinges in having some discrete conserved number of quasi-particles, and this produces a system of algebraic equations that describe scattering of these quasi-particles with each other and the boundary. The solutions of these Bethe equations give a finite number of roots that one identifies with the discrete spectrum of the finite system.

To have a continuous spectrum one needs one of two conditions: either the spin chain is infinite, or the conserved "number of quasiparticles" is described by a continuous parameter. The first case does not describe our system well, as we have found that the ground state has a well defined finite length (average number of spins). The second case would not produce algebraic equations that solve for the spectrum and we would be hard pressed to call such a system a Bethe Ansatz.

However we seem to have an integrable system with a continuous spectrum. We should contrast this observation with the observation of [27] that the Bethe Ansatz seems to break down at two loops for the maximal giant graviton, or that the BMN limit breaks down 28]. There are also other hints that the asymptotic Bethe Ansatz conjecture is incomplete [31. We interpret all of these facts not as a breakdown of integrability, but instead as a breakdown of applicability of a Bethe Ansatz.

\section{Discussion}

In this paper we have shown an example that dynamical finite D-branes can be treated consistently in the AdS/CFT setup. In particular we showed that we were able to characterize the D-brane in the most conservative form: as a geometric locus where open strings can end. We saw that this property could be derived exactly from the dual field theory. We were able to do this not just for a maximal giant graviton, but also for smaller giant gravitons that move in the AdS spacetime. The calculations required in field theory were rather subtle and we found a lot of surprises in trying to explain the dynamics of this system in detail.

The first surprise we found was that the trajectory of the spherical giants we studied contained families of null geodesics, and that it was possible to take a standard Penrose limit along these trajectories. We found that this limit gave us a different coordinate system than the usual one, because it was adapted to the D-brane being static.

We then found that is was possible in the limit of large quantum numbers to get the classical ratio of different components of angular momentum carried by the

ground state of the string. Part of it was along the direction of angular momentum of 
the giant, and the string and giant in principle exchange angular momentum between them.

We then looked for a description of these semiclassical states of the AdS geometry directly in the dual field theory in the perturbative weak coupling approximation. We found a spin chain model with variable numbers of sites at the boundary. We were also able to describe this system in terms of a boson chain model, where the individual bosons obeyed Cuntz algebra oscillator relations. The Hamiltonian we found was quadratic in generalized raising and lowering operators. It results as a limit $q \rightarrow 0$ of a $q$-deformed chain of harmonic oscillators with nearest neighbor interactions. The boson system did not preserve the total boson occupation number, so the boundary conditions we found are of non-diagonal type.

A big surprise was that when we tried to diagonalize the boson chain model we found a continuous spectrum of the effective hamiltonian. This was very unexpected when we started the project, but we found an analogy that made it intuitively obvious and necessary for the AdS/CFT correspondence to work. The analogy consists on thinking of the system as an open string whose ends are accelerated because they are attached to a D-brane, in the presence of a RR background. The background accelerates the D-brane, and the D-brane drags the string itself. The continuous spectrum was argued to be related to an instability that appears only if the string is too long and can not support its own weight. This instability might be interesting for the study of cosmic strings, because it can serve as a mechanism to seed long strings in an expanding universe.

We also found numerical evidence that the open string attached to the giant graviton was in general to one loop order governed by an integrable system. Our evidence is numerical and classical. We also found that the continuous spectrum seems to prevent the integrability of the system to be described by a Bethe Ansatz.

Nevertheless, despite this progress, there are many loose ends and open problems left to study. Here we would like to list some of them to encourage future research along these lines.

\section{The Plane Wave Spectrum and Integrability}

The issue of the integrability of the Hamiltonian (3.9) is important to prove the presence of the BMN spectrum. However, the fact that the spectrum has continuum energy bands suggests that integrability might be realized in a way quite different from the usual Heisenberg spin chain. In fact, since we have absorption and emission from the boundaries, one wonders if the usual formalism using the boundary reflection matrices could be generalized to take into account this effect [58]. Whatever the answer, this problem can have implications beyond the study of the AdS/CFT correspondence and should be very interesting to resolve. 


\section{The $D$-brane Instability}

As we mentioned before, the end state of the unstable giant graviton is difficult to predict from our current results. The reason is that all the approximations used in our field theory calculations are invalidated when the word's length describing the string is large with respect to $\sqrt{N}$. In any case, the end state of this instability can be important for the study of certain classical spacetimes. In particular we know that the $1 / 2$ BPS supergravity solutions found in [59] can be interpreted as coherent states of these giant gravitons (and the AdS giants). One can excite massive open string modes on these backgrounds and ask what effect the instability studied here can have on these backgrounds (one might imagine that these might serve as a mechanism to transport charge between droplets for example). This can be important for the stability of black holes since one can imagine that these will be constructed by exciting many open string modes on the D-branes. Finally, one can ask if this instability is also present in the case of AdS giant gravitons.

\section{Beyond the $S U(2)$ Sector}

In this paper we only considered open strings with two angular momenta on the sphere. In other words, an $S U(2)$ subsector of the corresponding gauge theory operators. However, it is interesting to go beyond this sector. At this moment we do not know how to write down a "nice" Hamiltonian describing the full $S O(6)$ excitations on the sphere. However, one can consider an $S U(3)$ subsector by using the three holomorphic fields: $X, Y$ and $Z$. The generalization of our Hamiltonian is straightforward. The most general word can be labeled as,

$$
\left(Y Z^{n_{1}} X Z^{n_{2}} Y \cdots Z^{n_{L}} X\right)_{i}^{j} \cong\left|s_{1}, n_{1}, s_{2}, n_{2}, \ldots, n_{L}, s_{L+1}\right\rangle
$$

where $s_{i}=\uparrow$ or $\downarrow \cong X$ or $Y$, is an $S U(2)$ spin label and $n_{i}$ is the usual bosonic occupation number.

The interactions between the sites are much the same as before except when any $n_{i}=0$. In this case we have the additional permutation interaction between the two spins $s_{i}$ and $s_{i+1}$ (see Eq. (C.14)). One can then write the Hamiltonian as,

$$
H=H_{\mathrm{boson}}+\lambda \sum_{l=1}^{L}\left(1-\hat{P}_{l, l+1}\right)\left(1-\hat{a}_{l}^{\dagger} \hat{a}_{l}\right),
$$

where $H_{\text {boson }}$ is the Hamiltonian for the bosonic lattice (3.9), and $\hat{P}_{l, l+1}=$ $\frac{1}{2}\left(1+4 \vec{S}_{l} \cdot \vec{S}_{l+1}\right)$ is the permutation operator acting on the spin sites. 
Constructing the action for the coherent states goes as usual (see appendix D). We get,

$$
\begin{aligned}
S= & -L \int d t \int_{0}^{1} d \sigma\left[\frac{r^{2}}{1-r^{2}} \dot{\phi}+\frac{1}{2} \cos \theta \dot{\varphi}+\frac{\lambda}{L^{2}}\left(r^{2}+r^{2} \phi^{\prime 2}\right)\right. \\
& \left.+\frac{\lambda}{4 L^{2}}\left(1-r^{2}\right)\left(\theta^{\prime 2}+\sin ^{2} \theta \varphi^{\prime 2}\right)\right],
\end{aligned}
$$

where the angles $\theta, \varphi$ label the $S U(2)$ coherent states. On the other hand, one can study the dual string theory and write the Polyakov action for open strings as we did in section 2. Using the natural coordinates adapted to the brane, and making the expansion in large total angular momentum in the $X$ and $Y$ directions we get the action (6.3) with the exception that the last term has a different $r$ dependence: $\sim \int d t d \sigma\left(1-r^{2}\right)^{2}\left(\theta^{\prime 2}+\sin ^{2} \theta \varphi^{\prime 2}\right)$. It would be interesting to understand the nature of this discrepancy.

\section{Multiple D-branes}

Recently it was shown how to write down the operators corresponding to multiple giant gravitons with strings attached to them [23]. In general the combinatorics are hopelessly complicated. However one can study the case of two giant gravitons with two strings stretching between them (we need two strings to satisfy the Gauss constraint [23]). Intuitively one would expect Hamiltonians like (3.9) but with more general coefficients for the boundary terms (different values at each boundary). In this case there can be a "current" of $Z$ fields from one giant to the other. It would be interesting to see what happens in this case. We expect that the giants attract each other. We would also expect the same instability we found for these models. Moreover, one should be able to measure the distance between the two giants using the sigma model representation of the coherent states. More generally, one should also be able to study D-branes at angles, and measure the angles between them by finding the spectrum of open strings stretching between them.

For many giants the easiest description seems to be in terms of the matrix model of [45]. However it is still not clear how to introduce the open string excitations in that language ${ }^{6}$. It would be interesting to re-derive the results presented in this paper using the matrix model formalism. This can also give a better understanding of the backreaction of the giant gravitons with massive open string modes.

\section{AdS Giants}

One can try to extend the results of this paper to the study of the giant gravitons that expand in the AdS space. The operators are similar to the

\footnotetext{
${ }^{6}$ See [26] for the emergence of closed string BMN excitations in the matrix model
} 
ones considered here but with the $\epsilon$ symbol replaced with totally symmetric tensor contractions. The combinatorics can be done in a similar way but the field theory interactions are more complicated when we consider an open string spinning along the AdS directions. This is because, instead of scalar "letters" we need to use covariant derivatives. However, one expects a simple description in terms of spin chains (or a bosonic lattice) as for closed strings [60, 10].

\section{Giants of critical angular momentum}

In this paper we have considered giant gravitons whose angular momentum grows proportionally to $N$. It would be interesting to study gravitons with angular momentum $p \sim \sqrt{N}$. For $p^{2} / N$ fixed and $p^{2} / N \ll 1$ the gravitons should be described as point-like supergravity modes (as it is done in the BMN limit). However, for $p^{2} / N$ fixed and $p^{2} / N \gg 1$ the appropriate description is in terms of expanded D3-branes. From (2.6) one immediately realizes that the angle $\theta_{0}$ goes now to zero in the large $N$ limit and each element of the giant is traveling in an almost null geodesic. Moreover, from (2.7) it is evident that the radius of the giant remains finite in the limit. Then, a short open string attached to this giant is effectively described by an open string attached to a spherical D3-brane of finite radius in a pp-wave background [61]. The string does not need to be spinning along the giant. Then, bosonic lattices with a finite and small number of sites should be used to describe the dilation operator in the dual field theory.

\section{Acknowledgements}

D.B. would like to thank A. Ludwig for conversations related to this work. D.B.and S.E.V. work was supported in part by a DOE award, under grant DE-FG02-91ER40618. D.H.C. work was supported by Fondecyt grant 3060009. Institutional grants to CECS of the Millennium Science Initiative, Fundación Andes, and support by Empresas CMPC are also acknowledged. S.E.V. work was supported in part by an NSF graduate fellowship.

\section{A. Penrose limit}

The Penrose limit is accomplished defining new coordinates by means of a linear transformation and taking an appropriate $R \rightarrow \infty$ limit. The coordinate playing the role of the curve parameter, must appear with the same coefficient in $t, \psi$ and $\eta$, if we want to capture the geometry near the null trajectory (2.9). The rest of the coefficients can be fixed by demanding the $R \rightarrow \infty$ limit to be well-defined. Then, 
we consider the transformation

$$
\begin{aligned}
t & =u+\frac{v}{R^{2}}, & \rho & =\frac{r}{R} \\
\psi & =u-\frac{v}{R^{2}}+\tan \theta_{0} \frac{x}{R}, & \theta & =\theta_{0}+\frac{y}{R} \\
\eta & =u-\frac{v}{R^{2}}-\cot \theta_{0} \frac{x}{R}, & \varphi & =\frac{z}{R \sin \theta_{0}} .
\end{aligned}
$$

After this scaling and the limit $R \rightarrow \infty$, the metric becomes,

$$
\begin{aligned}
d s^{2}= & -4 d u d v+4 y d u d x-\left(r^{2}+z^{2}\right) d u^{2} \\
& +d x^{2}+d y^{2}+d z^{2}+z^{2} d \xi^{2}+d r^{2}+r^{2} d \Omega_{3}^{2} .
\end{aligned}
$$

If we define cartesian coordinates

$$
\begin{array}{ll}
z_{1}=z \sin \xi, & z_{4}=r \sin \varphi^{\prime} \cos \eta^{\prime} \\
z_{2}=z \cos \xi, & z_{5}=r \cos \varphi^{\prime} \sin \xi^{\prime}, \\
z_{3}=r \sin \varphi^{\prime} \sin \eta^{\prime}, & z_{6}=r \cos \varphi^{\prime} \cos \xi^{\prime},
\end{array}
$$

the metric is written as,

$$
d s^{2}=-4 d u d v+4 y d u d x-\sum_{a=1}^{6} z_{a}^{2} d u^{2}+d x^{2}+d y^{2}+\sum_{a=1}^{6} d z_{a}^{2},
$$

and the RR 5-form field strength is

$$
F_{(5)}=2 d u \wedge\left(d z_{1} \wedge d z_{2} \wedge d z_{3} \wedge d z_{4}+d z_{5} \wedge d z_{6} \wedge d z_{7} \wedge d z_{8}\right)
$$

This pp-wave configuration can be put in the standard form using the following coordinate transformation 37.

$$
\begin{array}{ll}
x_{+}=u, & x_{1}=x \cos u+y \sin u, \\
x_{-}=v-\frac{1}{2} x y, & x_{2}=-x \sin u+y \cos u, \\
x_{a+2}=z_{a}, & \text { for } a=1, \ldots, 6 .
\end{array}
$$

which leads the metric to

$$
d s^{2}=-4 d x_{+} d x_{-}-\sum_{i=1}^{8} x_{i}^{2} d x_{+}^{2}+\sum_{i=1}^{8} d x_{i}^{2}
$$

Notice that this transformation involves rotations at constant angular velocity in the $x, y$ plane. In this sense, the D-brane we will be considering are rotating in the standard plane wave limit. 


\section{B. Brane in the Penrose limit}

To see how the D3-brane is specified in terms of the new coordinates (A.1) we should apply the coordinate transformation to the boundary conditions and then take the $R \rightarrow \infty$ limit. In the original coordinates, the giant expands in $(\varphi, \eta, \xi)$, and then for an open string, they should satisfy Neumann boundary conditions

$$
\begin{aligned}
& \left.\partial_{\sigma} \varphi\right|_{\sigma=0, \pi}=0 \\
& \left.\partial_{\sigma} \eta\right|_{\sigma=0, \pi}=0, \\
& \left.\partial_{\sigma} \xi\right|_{\sigma=0, \pi}=0 .
\end{aligned}
$$

On the other hand, the giant is situated in $\rho=0$ and $\theta=\theta_{0}$. Therefore, these coordinates have Dirichlet boundary conditions

$$
\begin{aligned}
& \left.\delta \rho\right|_{\sigma=0, \pi}=0, \\
& \left.\delta \theta\right|_{\sigma=0, \pi}=0 .
\end{aligned}
$$

The remaining transverse direction to the D-brane is $\psi$ and the D-brane is moving along this angle with $\psi=t$. The corresponding Dirichlet boundary condition is

$$
\left.\delta \psi\right|_{\sigma=0, \pi}=\left.\delta t\right|_{\sigma=0, \pi}
$$

As a consequence, the remaining Neumann boundary condition also mixes $t$ and $\psi$

$$
\left.\partial_{\sigma} t\right|_{\sigma=0, \pi}=\left.\cos ^{2} \theta_{0} \partial_{\sigma} \psi\right|_{\sigma=0, \pi}
$$

Using (A.1) the set of boundary conditions $($ B.1 $)-($ B.7 $)$ is translated, before the limit $R \rightarrow \infty$, into

$$
\begin{aligned}
\left.\partial_{\sigma} z\right|_{\sigma=0, \pi} & =0 \\
\left.\partial_{\sigma} \xi\right|_{\sigma=0, \pi} & =0, \\
\left.\partial_{\sigma} v\right|_{\sigma=0, \pi} & =0, \\
\left.\partial_{\sigma} u\right|_{\sigma=0, \pi} & =\left.\frac{\cot \theta_{0}}{R} \partial_{\sigma} x\right|_{\sigma=0, \pi}, \\
\left.\delta \rho\right|_{\sigma=0, \pi} & =0, \\
\left.\delta y\right|_{\sigma=0, \pi} & =0 \\
\left.\delta x\right|_{\sigma=0, \pi} & =\left.\frac{2 \cot \theta_{0}}{R} \delta v\right|_{\sigma=0, \pi} .
\end{aligned}
$$

So, in the $R \rightarrow \infty$ limit, we have Neumann boundary conditions for $u, v, z_{1}, z_{2}$ and Dirichlet boundary conditions for $x, y, z_{3}, z_{4}, z_{5}, z_{6}$. 


\section{Combinatorics and field theory calculations}

We begin by listing some properties of the totally anti-symmetric tensor that are useful for field theory calculations. This tensor is defined as

$$
\epsilon_{j_{1} \cdots j_{p}}^{i_{1} \cdots i_{p}} \equiv\left\{\begin{aligned}
1 & \text { if }\left(i_{1} \cdots i_{p}\right) \text { is an even permutation of }\left(j_{1} \cdots j_{p}\right) \\
-1 & \text { if }\left(i_{1} \cdots i_{p}\right) \text { is an odd permutation of }\left(j_{1} \cdots j_{p}\right) \\
0 & \text { otherwise }
\end{aligned}\right.
$$

where $p$ is any integer $p \leq N$, and $i_{1}, \cdots, i_{p}$ and $j_{1}, \cdots, j_{p}$ are integers from 1 to $N$. The simplest examples are

$$
\begin{aligned}
\epsilon_{j}^{i} & =\delta_{j}^{i}, \\
\epsilon_{k l}^{i j} & =\delta_{k}^{i} \delta_{l}^{j}-\delta_{l}^{i} \delta_{k}^{j} .
\end{aligned}
$$

Some of most useful properties of the $\epsilon$ tensor are

$$
\begin{aligned}
\epsilon_{j_{1} \cdots j_{p}}^{i_{1} \cdots i_{p}} & =\sum_{x=1}^{p}(-1)^{x+1} \delta_{j_{x}}^{i_{1}} \epsilon_{j_{1} \cdots j_{x-1} j_{x+1} \cdots j_{p}}^{i_{2}}, i_{p}^{i_{p}}, \\
\epsilon_{i_{1} \cdots i_{k} j_{k+1} \cdots j_{p}}^{i_{1} \cdots i_{k} i_{k+1} \cdots i_{p}} & =\frac{(N-p+k) !}{(N-p) !} \epsilon_{j_{k+1} \cdots j_{p}}^{i_{k+1} \cdots i_{p}}, \\
\epsilon_{j_{1} \cdots j_{k}}^{i_{1} \cdots i_{k}} \epsilon_{l_{1} \cdots l_{p}}^{j_{1} \cdots j_{p}} & =k ! \epsilon_{l_{1}}^{i_{1} \cdots i_{k} j_{k+1} \cdots j_{p}} \ldots l_{p} .
\end{aligned}
$$

Another useful identity that can be derived from the ones above is

$$
\epsilon_{i_{1} \cdots i_{p-1} \mu_{1}}^{j_{1} \cdots j_{p-1} \gamma_{1}} \epsilon_{j_{1} \cdots j_{p-1} \mu_{2} \cdots \mu_{k}}^{i_{1} \cdots i_{p-1} \gamma_{2} \cdots \gamma_{k}}=\frac{(p-1) !(N-k+1) !}{(N-p-k+2) !}\left(\delta_{\mu_{1}}^{\gamma_{1}} \epsilon_{\mu_{2} \cdots \mu_{k}}^{\gamma_{2} \cdots \gamma_{k}}-\frac{(p-1)}{(N-k+1)} \epsilon_{\mu_{1} \cdots \mu_{k}}^{\gamma_{1} \cdots \gamma_{k}}\right) .
$$

In the rest of this appendix we present some of the field theory calculations leading to the Hamiltonian (3.9). Most of the calculations are very similar to those presented in [25] in the context of maximal giant gravitons.

We begin by pointing out that an operator with a $Z$ field at the end or beginning of the word does not represent a linearly independent state. In fact, from the identities above one can show that

$$
\epsilon_{i_{1} \cdots i_{p}}^{j_{1} \cdots j_{p}} Z_{j_{1}}^{i_{1}} \cdots Z_{j_{p-1}}^{i_{p-1}}(Z W)_{j_{p}}^{i_{p}}=\frac{1}{p} \epsilon_{i_{1} \cdots i_{p}}^{j_{1} \cdots j_{p}} Z_{j_{1}}^{i_{1}} \cdots Z_{j_{p}}^{i_{p}} \operatorname{Tr}(W)-\frac{1}{p} \epsilon_{i_{1} \cdots i_{p+1}}^{j_{1} \cdots j_{p+1}} Z_{j_{1}}^{i_{1}} \cdots Z_{j_{p}}^{i_{p}} W_{j_{p+1}}^{i_{p+1}} .
$$

This shows that the operator with a $Z$ at the border of the word factorizes into a brane with a closed string and a bigger brane.

Now we turn our attention to the correlation functions. These will be computed to one loop in the 't Hooft coupling and using the planar approximation of the large 
$N$ limit. We will be using the bosonic part of the SYM action given by

$$
S=\frac{1}{2 \pi g_{s}} \int d^{4} x \operatorname{Tr}\left(\frac{1}{2} F_{\mu \nu} F^{\mu \nu}+D_{\mu} X D^{\mu} \bar{X}+D_{\mu} Y D^{\mu} \bar{Y}+D_{\mu} Z D^{\mu} \bar{Z}+V_{D}+V_{F}\right),
$$

where,

$$
\begin{aligned}
& V_{D}=\frac{1}{2} \operatorname{Tr}\left(|[X, \bar{X}]+[Y, \bar{Y}]+[Z, \bar{Z}]|^{2}\right), \\
& V_{F}=2 \operatorname{Tr}\left(|[X, Y]|^{2}+|[X, Z]|^{2}+|[Y, Z]|^{2}\right) .
\end{aligned}
$$

Let us begin with the free field correlation functions, since they are going to give us the normalization of the operators. The propagator for the scalars is of the form

$$
\left\langle\phi_{j}^{i}(x) \bar{\phi}_{l}^{k}(0)\right\rangle=\frac{g_{s}}{2 \pi} \frac{1}{|x|^{2}} \delta_{l}^{i} \delta_{j}^{k}
$$

where $\phi$ is any of the complex scalars $X, Y, Z$. Thus we see that the correlation function of any word with a total classical dimension $\Delta_{0}$ will be multiplied by the following overall numerical factor: $\left(\frac{g_{s}}{2 \pi} \frac{1}{|x|^{2}}\right)^{\Delta_{0}}$. In what follows we will drop this factor from the calculations for simplicity.

Let us now compute the correlation function for the operator

$$
\mathcal{O}_{W}^{p}=\epsilon_{i_{1} \cdots i_{p}}^{j_{1} \cdots j_{p}} Z_{j_{1}}^{i_{1}} \cdots Z_{j_{p-1}}^{i_{p-1}} W_{j_{p}}^{i_{p}}
$$

where the word $W$ has classical dimension $L$. We will assume that the operator is very long such that $p>\sqrt{N}$ and $L \lesssim \sqrt{N}$ with $p \gg L$. In the large $N$ limit, the leading contribution will come from contracting all the $Z$ fields and then the words planarly. Moreover, in the large $N$ limit operators with different values of $p$ and word lengths will be orthogonal. Thus the free theory two point function gives

$$
\begin{aligned}
\left\langle\overline{\mathcal{O}}_{W}^{p} \mathcal{O}_{W}^{p}\right\rangle_{\mathrm{free}} & \sim(p-1) ! \epsilon_{i_{1} \cdots i_{p-1} \mu_{1}}^{j_{1} \cdots j_{p-1} \gamma_{1}} \epsilon_{j_{1} \cdots j_{p-1} \mu_{2}}^{i_{1} \cdots i_{p-1} \gamma_{2}}\left\langle\bar{W}_{\gamma_{1}}^{\mu_{1}} W_{\gamma_{2}}^{\mu_{2}}\right\rangle_{\mathrm{free}} \\
& =\frac{(p-1) !^{2}(N-2) !}{(N-p-1) !}\left[\langle\operatorname{Tr}(\bar{W}) \operatorname{Tr}(W)\rangle_{\mathrm{free}}+\frac{(p-1)}{N-p}\langle\operatorname{Tr}(\bar{W} W)\rangle_{\text {free }}\right] \\
& \sim \frac{p !(p-1) !(N-1) ! N^{L}}{(N-p) !}
\end{aligned}
$$

In going to the second line of $(\mathrm{C.13})$ we used the identity $(\overline{\mathrm{C} .6})$ and for the third line we contracted the words using the planar approximation.

If the word $W$ is made of a single type of field we will get an additional multiplicative factor of $L(1+\mathcal{O}(L / N))$ to (C.13) from the cyclic property of the trace. This is one of the reasons we consider only $L \ll N$ and in particular $L \lesssim \sqrt{N}$.

We now turn on the Yang-Mills coupling and consider the interactions between the scalars. Let us start by calculating the interactions in the bulk of the words. This 
terms are obtained when all the $Z$ fields of the giants are contracted we free field propagators and the vertex is entirely contracted with letters of the words. In the planar approximation these will be the familiar nearest-neighbor interactions that were considered by Minahan and Zarembo [9]. Thus if we regard each "letter" in $W$ as a $S O(6)$ vector, the nearest-neighbor interactions have the familiar form,

$$
H_{l, l+1}=\frac{1}{2} \lambda\left[K_{l, l+1}+2\left(I_{l, l+1}-P_{l, l+1}\right)\right],
$$

where $K$ and $P$ are the trace and permutation operators respectively and $\lambda=$ $g_{s} N / 2 \pi$. For the $S U(2)$ and $S U(3)$ sectors considered above, the trace will be zero and it is easy to see that the remaining interactions account for the first two terms in the Hamiltonian (3.9).

For completeness, let us show how to calculate the bulk part of the Hamiltonian by considering the example of the interactions giving rise to the identity in (C.14). To this end we study the interaction between a $X$ and $Y$ fields of the operator

$$
\mathcal{O}_{X Y}^{p}=\epsilon_{i_{1} \cdots i_{p}}^{j_{1} \cdots j_{p}} Z_{j_{1}}^{i_{1}} \cdots Z_{j_{p-1}}^{i_{p-1}}\left(W_{1} X Y W_{2}\right)_{j_{p}}^{i_{p}} .
$$

It is well known that for chiral operators the interactions coming from $V_{D}$ cancel with the gauge boson exchange and the scalar self energies. Therefore, the only relevant interactions between $X$ and $Y$ will come from the $\mathrm{F}$ term. It is not difficult to see that the only planar interaction will be,

$$
-\frac{1}{\pi g_{s}} \int d^{4} x \operatorname{Tr}(X Y \bar{Y} \bar{X}) .
$$

This interaction is illustrated in figure 7 .

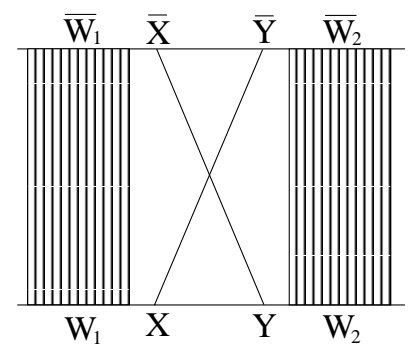

Figure 7: One-loop interaction between two nearest-neighbor letters of a word generated by a vertex of the form $\operatorname{Tr}(X Y \bar{Y} \bar{X})$.

The rest of the free theory contractions go as before. Therefore, all we get from the interaction is a multiplicative correction to (C.13) of the form

$$
\left(\frac{g_{s}}{2 \pi}\right)^{-2}\left(\frac{g_{s}}{2 \pi}\right)^{4} N\left(-\frac{1}{\pi g_{s}}\right)|x|^{4} \int d^{4} y \frac{1}{|y|^{4}|x-y|^{4}} \approx-2 \lambda \log (|x| \Lambda) .
$$


Thus, after dividing by the norm (C.13) and using the definition of the anomalous dimension matrix (3.5), we see that this interaction will give a numerical contribution of $\lambda$ to the identity interaction in agreement with (C.14).

Now let us see what happens when we have a non-chiral operator. Let us consider the word $W_{1} X \bar{Y} W_{2}$. In this case the D-term interaction will no longer cancel with the gauge boson exchange and the scalar self-energies. However, we can exploit the fact that these last two interactions are flavor blind to write their contribution in terms of the D-term contribution to the chiral operator. This is illustrated in figure 8.

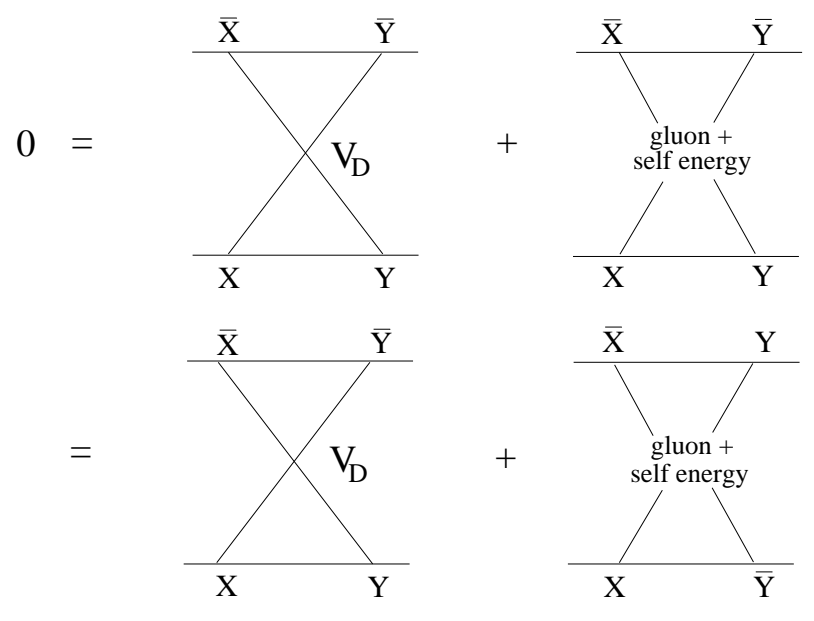

Figure 8: The first line of the figure illustrates the cancelation of the D-term contribution with the gluon exchange and the scalars self energies for a chiral operator. Since the gluon and self energy interactions are flavor blind they will give the same numerical value if we compute them using the non-chiral operator as in the second line.

It is not difficult to see that there is no planar contribution to the identity coming from the F-term. Thus, the total contribution to this interaction is from the diagrams shown in figure 9 .

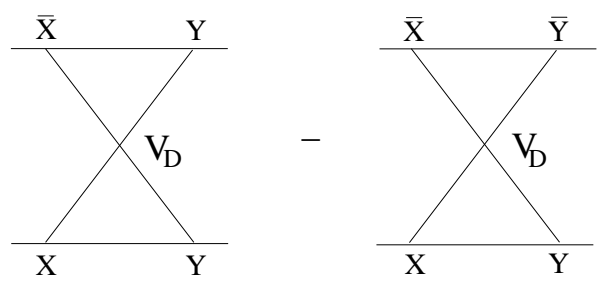

Figure 9: Total contribution to the one-loop interaction between $X$ and $\bar{Y}$ of the word $W_{1} X \bar{Y} W_{2}$. The second term is the gluon and self energy interactions calculated in terms of $V_{D}$ using the result illustrated in the previous figure. 
The first diagram comes from the vertex

$$
-\frac{1}{2 \pi g_{s}} \operatorname{Tr}(X \bar{Y} Y \bar{X})
$$

and the second from

$$
-\frac{1}{2 \pi g_{s}} \operatorname{Tr}(X Y \bar{Y} \bar{X})
$$

Note that the second vertex is to be evaluated between the chiral operator. At the end, we see that we will get a numerical contribution of $\lambda / 2$ from each interaction, and this will sum up to the contribution shown in (C.14). The rest of the interactions in the Hamiltonian (C.14) can be obtained in a similar way.

Let us now turn our attention to the interactions between the word and the $Z$ fields of the giant graviton. The first contribution comes from the scalars at the ends of the word. Thus we can study the operator

$$
\mathcal{O}_{\phi}^{p}=\epsilon_{i_{1} \cdots i_{p}}^{j_{1} \cdots j_{p}} Z_{j_{1}}^{i_{1}} \cdots Z_{j_{p-1}}^{i_{p-1}}(\phi W)_{j_{p}}^{i_{p}}
$$

where $\phi$ is any of the complex scalars except $Z$ and the word $W$ has length $L-1$. Doing the free contractions we get

$$
\begin{aligned}
\left\langle\overline{\mathcal{O}}_{\phi}^{p}(x) \mathcal{O}_{\phi}^{p}(0)\right\rangle \sim & N^{L-2}(p-1)^{2}(p-2) ! \epsilon_{i_{1} \cdots i_{p-2} \mu_{1} \mu_{2}}^{j_{1} \cdots j_{p-2} \gamma_{1} \gamma_{2}} \epsilon_{j_{1} \cdots j_{p-2} \mu_{3} \gamma_{2}}^{i_{1} \cdots i_{p-2} \gamma_{3} \gamma_{4}} \\
& \times\left\langle\bar{Z}_{\gamma_{1}}^{\mu_{1}}(x) Z_{\gamma_{3}}^{\mu_{3}}(0)[\phi(0) \bar{\phi}(x)]_{\gamma_{4}}^{\mu_{2}}\right\rangle .
\end{aligned}
$$

We now note that there are only two relevant planar interactions. These will have the general form:

$$
-\frac{\alpha}{\pi g_{s}} \operatorname{Tr}(Z \bar{Z} \phi \bar{\phi}), \quad-\frac{\beta}{\pi g_{s}} \operatorname{Tr}(\bar{Z} Z \phi \bar{\phi}) .
$$

If the field $\phi$ is $\bar{X}, \bar{Y}$ or $\bar{Z}$, the constants $\alpha, \beta$ will need to include the contribution from the gauge boson exchange and the scalar self energies. In any case, the interactions (C.22) will give a contribution to (C.21) of

$$
\left\langle\overline{\mathcal{O}}_{\phi}^{p}(x) \mathcal{O}_{\phi}^{p}(0)\right\rangle \sim-2 \lambda N^{L} \frac{p !(p-1) !(N-1) !}{(N-p) !}\left[\alpha \frac{p}{N}+\beta\left(1-\frac{p}{N}\right)\right] \log (|x| \Lambda),
$$

up to multiplicative corrections of order $1 / p$ and $1 / N$. Thus after dividing by the norm (C.13) we get the following contribution to the anomalous dimension: $\lambda[\alpha p / N+\beta(1-p / N)]$. Calculating the constants $\alpha$ and $\beta$ is just a matter of counting how many terms like (C.22) we can find in $V_{D}$ and $V_{F}$. At the end we get that for the fields $X, Y, \bar{X}$ or $\bar{Y}, \alpha=0$ and $\beta=1$. Thus the anomalous dimension gives $\lambda(1-p / N)$. Translating to the boson lattice language and taking into account the 
contribution to the field at the other end of the word, this gives the first term in the second line of the Hamiltonian (3.9). On the other hand, if $\phi=\bar{Z}$ we have that $\alpha=-1$ and $\beta=3 / 2$. Thus the contribution to the anomalous dimension is $\lambda(1+(1-p / N) / 2)$.

Finally, there is an interaction that is not present in the case of the maximal giant graviton. This is the exchange of a $Z$ field between the word and the giant graviton. In the planar approximation this can only happen when the $Z$ is the second or next to last letter of the word. To calculate this amplitude we can consider the correlation between the operators

$$
\mathcal{O}^{p+1}=\epsilon_{i_{1} \cdots i_{p+1}}^{j_{1} \cdots j_{p+1}} Z_{j_{1}}^{i_{1}} \cdots Z_{j_{p}}^{i_{p}}(X W)_{j_{p+1}}^{i_{p+1}}, \quad \mathcal{O}^{p}=\epsilon_{i_{1} \cdots i_{p}}^{j_{1} \cdots j_{p}} Z_{j_{1}}^{i_{1}} \cdots Z_{j_{p-1}}^{i_{p-1}}(X Z W)_{j_{p}}^{i_{p}}
$$

and assume that the word $W$ has classical dimension $L$. The calculation here is very similar to the previous example. The free contractions give

$$
\left\langle\overline{\mathcal{O}}^{p+1}(x) \mathcal{O}^{p}(0)\right\rangle \sim N^{L-1} p ! \epsilon_{i_{1} \cdots i_{p-1} \mu_{1} \mu_{2}}^{j_{1} \cdots j_{p-1} \gamma_{1}} \epsilon_{j_{1} \cdots j_{p-1} \gamma_{2}}^{i_{1} \cdots i_{p-1} \gamma_{3}}\left\langle\bar{Z}_{\gamma_{1}}^{\mu_{1}}(X Z \bar{X})_{\gamma_{3}}^{\mu_{2}}\right\rangle
$$

Since these are chiral operators, the only interactions will come from $V_{F}$. It is easy to see that the only planar interaction will be

$$
+\frac{1}{\pi g_{s}} \operatorname{Tr}(Z X \bar{Z} \bar{X})
$$

Doing the contractions we get

$$
\begin{aligned}
\left\langle\overline{\mathcal{O}}^{p+1}(x) \mathcal{O}^{p}(0)\right\rangle & \sim 2 \lambda N^{L} p ! \epsilon_{i_{1} \cdots i_{p-1} \mu_{1} \gamma_{1}}^{j_{1} \cdots j_{p-1} \gamma_{1} \gamma_{2}} \epsilon_{j_{1} \cdots j_{p-1} \gamma_{2}}^{i_{1} \cdots i_{p-1} \mu_{1}} \log (|x| \Lambda) \\
& =-2 \lambda N^{L+2} \frac{p !^{2}(N-1) !}{(N-p) !}\left(1-\frac{p}{N}\right) \log (|x| \Lambda),
\end{aligned}
$$

where we have used the identities (C.4) and (C.5).

The norms are

$$
\begin{aligned}
\left\langle\overline{\mathcal{O}}^{p} \mathcal{O}^{p}\right\rangle_{\text {free }} & \sim \frac{p !^{2}(N-1) ! N^{L+2}}{p(N-p) !}, \\
\left\langle\overline{\mathcal{O}}^{p+1} \mathcal{O}^{p+1}\right\rangle_{\text {free }} & \sim \frac{(p+1) p !^{2}(N-1) ! N^{L+2}}{(N-p) !}\left(1-\frac{p}{N}\right) .
\end{aligned}
$$

Therefore, for large $p$ the contribution to the anomalous dimension is: $\lambda \sqrt{1-p / N}$. We recognize this as sources/sinks in the Hamiltonian (3.9).

To conclude we show that closed string emission/absorption is suppressed in the large $N$ limit if we take $p \sim \gamma N$. For definitiveness consider the operators

$$
\mathcal{O}_{1}^{p}=\epsilon_{i_{1} \cdots i_{p}}^{j_{1} \cdots j_{p}} Z_{j_{1}}^{i_{1}} \cdots Z_{j_{p}}^{i_{p}} \operatorname{Tr}\left(Y^{L}\right), \quad \mathcal{O}_{2}^{p}=\epsilon_{i_{1} \cdots i_{p}}^{j_{1} \cdots j_{p}} Z_{j_{1}}^{i_{1}} \cdots Z_{j_{p-1}}^{i_{p-1}}\left(Y Z Y^{L-1}\right)_{j_{p}}^{i_{p}}
$$


Then, the interacting correlation function gives (up to signs or numerical factors)

$$
\left\langle\overline{\mathcal{O}}_{1}^{p} \mathcal{O}_{2}^{p}\right\rangle \sim \lambda \frac{L p !^{2}(N-1) ! N^{L}}{(N-p) !} \log (|x| \Lambda),
$$

The norms are,

$$
\left\langle\overline{\mathcal{O}}_{1} \mathcal{O}_{1}\right\rangle_{\text {free }} \sim \frac{L p !^{2} N ! N^{L}}{(N-p) !}, \quad\left\langle\overline{\mathcal{O}}_{2} \mathcal{O}_{2}\right\rangle_{\text {free }} \sim \frac{p !^{2} N ! N^{L}}{p(N-p) !},
$$

Therefore, the contribution to the anomalous dimension is of the order

$$
\lambda \frac{\sqrt{L p}}{N} \sim \lambda \sqrt{\frac{L}{N}},
$$

This is clearly suppressed in the limit we are considering of $L \lesssim \sqrt{N}$.

\section{Calculations in the $S U(3)$ sector}

Here we give some more details about the calculation of the effective sigma model action for the $S U(3)$ sector. We begin with the gauge theory side. The coherent states for states of the form (6.1) take the product form

$$
|\mathrm{CS}\rangle=\bigotimes_{i=1}^{L}\left|\vec{n}_{i}, z_{i}\right\rangle \otimes\left|\vec{n}_{L+1}\right\rangle,
$$

where $\left|z_{i}\right\rangle$ are the bosonic coherent states $(\mathbb{E} .2)$ and $\left|\vec{n}_{i}\right\rangle$ are the $S U(2)$ coherent states defined by [53, 32],

$$
|\vec{n}\rangle=e^{i S_{z} \varphi} e^{i S_{y} \theta}|\uparrow\rangle,
$$

where $\vec{n}=(\sin \theta \cos \varphi, \sin \theta \sin \varphi, \cos \theta)$ is a unit vector in $S^{2}$. They obey

$$
\begin{gathered}
\langle\vec{n}|\vec{S}| \vec{n}\rangle=\frac{1}{2} \vec{n} \\
\left\langle\vec{n} \mid \vec{n}^{\prime}\right\rangle=\left[\cos \frac{1}{2}\left(\theta-\theta^{\prime}\right) \cos \frac{1}{2}\left(\varphi-\varphi^{\prime}\right)-i \cos \frac{1}{2}\left(\theta+\theta^{\prime}\right) \sin \frac{1}{2}\left(\theta-\theta^{\prime}\right)\right] e^{i \frac{1}{2}\left(\varphi-\varphi^{\prime}\right)} .
\end{gathered}
$$

The action for the coherent states is

$$
S=\lim _{L \rightarrow \infty} \int d t\left(i\left\langle\mathrm{CS}\left|\partial_{t}\right| \mathrm{CS}\right\rangle-\langle\mathrm{CS}|H| \mathrm{CS}\rangle\right)
$$

and we get (6.3). 
On the other hand, one can study the dual string theory and write the Polyakov action for open strings as we did in section 2. In the case of three spins, the natural coordinates on the sphere $|X|^{2}+|Y|^{2}+|Z|^{2}=1$ are

$$
X= \pm \sqrt{1-r^{2}} \cos \theta e^{i \varphi_{1}}, \quad Y= \pm \sqrt{1-r^{2}} \sin \theta e^{i \varphi_{2}}, \quad Z=r e^{i(t-\phi)}
$$

where the giant graviton is located at $r=\sqrt{1-p / N}$ and $\varphi=\varphi_{1}+\varphi_{2}-t$. Following [34] we can make another change of coordinates:

$$
\alpha=\frac{1}{2}\left(\varphi_{1}+\varphi_{2}\right), \quad \beta=\frac{1}{2}\left(\varphi_{1}-\varphi_{2}\right), \quad U_{1}=\cos \theta e^{i \beta}, \quad U_{2}=\sin \theta e^{-i \beta}
$$

In these coordinates, the metric on $\mathbb{R} \times S^{5}$ takes the form

$$
d s^{2}=-\left(1-r^{2}\right) d t^{2}+\frac{1}{1-r^{2}} d r^{2}+r^{2} d \phi^{2}+2 r^{2} d \phi d t+\left(1-r^{2}\right)\left[(D \alpha)^{2}+\frac{1}{4} d n_{i} d n_{i}\right]
$$

where coordinates on a $S^{2}$ are given by $n_{i}=\xi^{\dagger} \sigma_{i} \xi$, where $\xi=\left(U_{1} e^{i \alpha}, U_{2} e^{-i \alpha}\right)$ and $\sigma_{i}$ are the Pauli matrices. The covariant derivative is defined as $D \alpha=d \alpha+C$, where $C=-i \bar{U}_{i} d U_{i}$.

We now want to expand the Polyakov action in the limit of large angular momentum in $\alpha$, and choose a gauge where this angular momentum is uniformly distributed along the string. Note that this angular momentum is dual to the total number of $X \mathrm{~s}$ and $Y \mathrm{~s}$ in the word (6.1). We choose the coordinates in the Polyakov action such that $\alpha$ is coupled through its covariant derivatives $\sim g^{a b} D_{a} \alpha D_{b} \alpha$, where $D_{a} \alpha=\partial_{a} \alpha-i \bar{U}_{i} \partial_{a} U_{i}$

With this choice, the lagrangian density in momentum space (2.37) becomes

$$
\mathcal{L}=p_{t} \dot{t}+p_{r} \dot{r}+p_{\phi} \dot{\phi}+p_{\alpha} D_{t} \alpha+p_{i} \dot{n}_{i}
$$

and we have the usual constraints:

$$
\begin{aligned}
G^{\mu \nu} p_{\mu} p_{\nu}+G_{\mu \nu} X^{\prime \mu} X^{\prime \nu} & =0 \\
p_{\mu} X^{\prime \mu} & =0
\end{aligned}
$$

We then choose the usual gauge: $p_{\alpha}=2 \mathcal{J}=$ constant and $t=\tau$, where the total angular momentum in $\alpha$ is $L=\sqrt{\lambda_{Y M}} \mathcal{J}$. From (D.11) we can eliminate $D_{1} \alpha$ and then use $(\mathbb{D . 1 0})$ to eliminate $p_{t}$ from the Lagrangian $(\mathbb{D . 9})$. The result is

$$
\mathcal{L}=p_{\phi}+p_{a} \dot{X}^{a}+2 \mathcal{J} C_{0}-\sqrt{\Lambda^{a b} p_{a} p_{b}+\Lambda}
$$


where the indices $a, b$ run trough the rest of the coordinates excluding $t$ and $\alpha$. The functions $\Lambda^{a b}$ and $\Lambda$ are

$$
\begin{aligned}
\Lambda & =\frac{4 \mathcal{J}^{2}}{1-r^{2}}+\frac{r^{\prime 2}}{1-r^{2}}+r^{2} \phi^{2}+\left(1-r^{2}\right) n_{i}^{\prime 2}, \\
\Lambda^{r r} & =\left(1-r^{2}\right)\left(1+\frac{r^{\prime 2}}{4 \mathcal{J}^{2}}\right), \\
\Lambda^{\phi \phi} & =1+\left(1-r^{2}\right)\left(\frac{1}{r^{2}}+\frac{\phi^{\prime 2}}{4 \mathcal{J}^{2}}\right), \\
\Lambda^{i i} & =\frac{1}{1-r^{2}}+\frac{1-r^{2}}{4 \mathcal{J}^{2}} n_{i}^{\prime 2} \\
\Lambda^{r \phi} & =\Lambda^{\phi r}=\frac{\left(1-r^{2}\right) r^{\prime} \phi^{\prime}}{4 \mathcal{J}^{2}} \\
\Lambda^{r i} & =\Lambda^{i r}=\frac{\left(1-r^{2}\right) r^{\prime} n_{i}^{\prime}}{4 \mathcal{J}^{2}} \\
\Lambda^{\phi i} & =\Lambda^{i \phi}=\frac{\left(1-r^{2}\right) \phi^{\prime} n_{i}^{\prime}}{4 \mathcal{J}^{2}} \\
\Lambda^{i j} & =\Lambda^{j i}=\frac{\left(1-r^{2}\right) n_{i}^{\prime} n_{j}^{\prime}}{4 \mathcal{J}^{2}} .
\end{aligned}
$$

We then proceed as before by eliminating the momenta using their equations of motion. This gives

$$
\mathcal{L}=2 \mathcal{J} C_{0}-\sqrt{\Lambda\left(1-\Lambda_{a b} \dot{x}^{a} \dot{x}^{b}\right)},
$$

where $\dot{x}^{a}=\dot{X}^{a}$ for $a \neq \phi$ and $\dot{x}^{\phi}=1+\dot{\phi}$, and $\Lambda_{a b}$ is the inverse of the symmetric matrix $\Lambda^{a b}$.

One can now expand as usual in the limit of large $\mathcal{J}$ assuming $\dot{X}^{a} \sim \mathcal{O}\left(1 / \mathcal{J}^{2}\right)$. To lowest non-trivial order we get the action (6.3) except that for the $r$ dependence of the last term we get $\int d^{2} \sigma\left(1-r^{2}\right)^{2}\left(\partial_{1} n_{i}\right)^{2}$.

\section{E. Coherent states for the $q$-deformed algebra}

The $q$-deformed algebra has the following representation [62],

$$
a^{\dagger}|n\rangle=\sqrt{[n+1]}|n+1\rangle, \quad a|n\rangle=\sqrt{[n]}|n-1\rangle, \quad \text { with }[n] \equiv \frac{1-q^{n}}{1-q} .
$$

Coherent states can be defined as eigenstates of the annihilation operator, and they are given by 63

$$
|z\rangle=\left(\exp _{q}\left(|z|^{2}\right)\right)^{-1 / 2} \sum_{n=0}^{\infty} \frac{z^{n}}{\sqrt{[n] !}}|n\rangle, \quad \text { with } \quad|z|<\frac{1}{1-q},
$$


where $[n] !=[1] .[2] \ldots[n]$. The $q$-exponential is defined by,

$$
\exp _{q}(x)=\sum_{n=0}^{\infty} \frac{x^{n}}{[n] !}=\frac{1}{\prod_{k=0}^{\infty}\left(1-q^{k}(1-q) x\right)}
$$

As usual, these coherent states constitute a non-orthogonal and overcomplete basis. The overlapping between coherent states is

$$
\left\langle z \mid z^{\prime}\right\rangle=\frac{\exp _{q}\left(\bar{z} z^{\prime}\right)}{\left(\exp _{q}\left(|z|^{2}\right) \exp _{q}\left(\left|z^{\prime}\right|^{2}\right)\right)^{1 / 2}} .
$$

The resolution of the identity takes the form,

$$
\frac{1}{\pi} \int_{D_{q}} d_{q}^{2} z|z\rangle\left\langle z\left|=\frac{1}{2 \pi} \int_{0}^{2 \pi} d \phi \int_{0}^{1 /(1-q)^{2}} d_{q}\left(r^{2}\right)\right| z\right\rangle\langle z|=1
$$

where $D_{q}$ is the open disk in the complex plane with radius $1 /(1-q), z=r e^{i \phi}$ and the integral over $r$ is the so-called Jackson Integral,

$$
\int_{0}^{a} f(x) d_{q} x=a(1-q) \sum_{n=0}^{\infty} q^{k} f\left(q^{k} a\right)
$$

which satisfies $\int_{0}^{x_{1}}\left(\exp _{q}(x)\right)^{-1} x^{n} d_{q} x=[n]$ !.

\section{References}

[1] G. 't Hooft, "A Planar Diagram Theory For Strong Interactions," Nucl. Phys. B 72, 461 (1974).

[2] J. M. Maldacena, "The large N limit of superconformal field theories and supergravity," Adv. Theor. Math. Phys. 2, 231 (1998) [Int. J. Theor. Phys. 38, 1113 (1999)] [arXiv:hep-th/9711200].

[3] E. Witten, "Anti-de Sitter space and holography," Adv. Theor. Math. Phys. 2, 253 (1998) [arXiv:hep-th/9802150].

[4] S. S. Gubser, I. R. Klebanov and A. M. Polyakov, "Gauge theory correlators from non-critical string theory," Phys. Lett. B 428, 105 (1998) [arXiv:hep-th/9802109].

[5] M. Blau, J. Figueroa-O'Farrill, C. Hull and G. Papadopoulos, "A new maximally supersymmetric background of IIB superstring theory," JHEP 0201, 047 (2002) [arXiv:hep-th/0110242].

[6] R. R. Metsaev, "Type IIB Green-Schwarz superstring in plane wave Ramond-Ramond background," Nucl. Phys. B 625, 70 (2002) [arXiv:hep-th/0112044]. 
[7] M. Blau, J. Figueroa-O'Farrill, C. Hull and G. Papadopoulos, "Penrose limits and maximal supersymmetry," Class. Quant. Grav. 19, L87 (2002) [arXiv:hep-th/0201081].

[8] D. Berenstein, J. M. Maldacena and H. Nastase, "Strings in flat space and pp waves from N = 4 super Yang Mills," JHEP 0204, 013 (2002) [arXiv:hep-th/0202021].

[9] J. A. Minahan and K. Zarembo, ' "The Bethe-ansatz for N = 4 super Yang-Mills," JHEP 0303 (2003) 013. [arXiv:hep-th/0212208].

[10] N. Beisert and M. Staudacher, "The N = 4 SYM integrable super spin chain," Nucl. Phys. B 670, 439 (2003) [arXiv:hep-th/0307042].

[11] I. Bena, J. Polchinski and R. Roiban, "Hidden symmetries of the AdS(5) x S**5 superstring," Phys. Rev. D 69, 046002 (2004) [arXiv:hep-th/0305116].

[12] L. Dolan, C. R. Nappi and E. Witten, "A relation between approaches to integrability in superconformal Yang-Mills theory," JHEP 0310, 017 (2003) [arXiv:hep-th/0308089].

[13] D. Berenstein and S. A. Cherkis, "Deformations of N = 4 SYM and integrable spin chain models," Nucl. Phys. B 702, 49 (2004) [arXiv:hep-th/0405215].

[14] O. DeWolfe and N. Mann, "Integrable open spin chains in defect conformal field theory," JHEP 0404, 035 (2004) [arXiv:hep-th/0401041].

[15] B. Chen, X. J. Wang and Y. S. Wu, "Integrable open spin chain in super Yang-Mills and the plane-wave / SYM duality," JHEP 0402, 029 (2004) [arXiv:hep-th/0401016].

[16] B. Chen, X. J. Wang and Y. S. Wu, "Open spin chain and open spinning string," Phys. Lett. B 591, 170 (2004) [arXiv:hep-th/0403004].

[17] J. McGreevy, L. Susskind and N. Toumbas, "Invasion of the giant gravitons from anti-de Sitter space," JHEP 0006, 008 (2000) [arXiv:hep-th/0003075].

[18] V. Balasubramanian, M. Berkooz, A. Naqvi and M. J. Strassler, "Giant gravitons in conformal field theory," JHEP 0204, 034 (2002) [arXiv:hep-th/0107119].

[19] S. Corley, A. Jevicki and S. Ramgoolam, "Exact correlators of giant gravitons from dual N = 4 SYM theory," Adv. Theor. Math. Phys. 5, 809 (2002) [arXiv:hep-th/0111222].

[20] D. Berenstein, "A toy model for the AdS/CFT correspondence," JHEP 0407, 018 (2004) [arXiv:hep-th/0403110].

[21] D. Berenstein, C. P. Herzog and I. R. Klebanov, "Baryon spectra and AdS/CFT correspondence," JHEP 0206, 047 (2002) [arXiv:hep-th/0202150].

[22] V. Balasubramanian, M. x. Huang, T. S. Levi and A. Naqvi, "Open strings from N = 4 super Yang-Mills,” JHEP 0208, 037 (2002) [arXiv:hep-th/0204196]. 
[23] D. Berenstein, "Shape and holography: Studies of dual operators to giant gravitons," Nucl. Phys. B 675 (2003) 179 [arXiv:hep-th/0306090].

[24] V. Balasubramanian, D. Berenstein, B. Feng and M. x. Huang, "D-branes in Yang-Mills theory and emergent gauge symmetry," [arXiv:hep-th/0411205].

[25] D. Berenstein and S. E. Vazquez, "Integrable open spin chains from giant gravitons," JHEP 0506, 059 (2005) [arXiv:hep-th/0501078].

[26] D. Berenstein, D. H. Correa and S. E. Vazquez, "Quantizing open spin chains with variable length: An example from giant gravitons," Phys. Rev. Lett. 95, 191601 (2005) [arXiv:hep-th/0502172].

[27] A. Agarwal, "Open spin chains in super Yang-Mills at higher loops: Some potential problems with integrability," arXiv:hep-th/0603067.

[28] K. Okamura and K. Yoshida, "Higher Loop Bethe Ansatz for Open Spin-Chains in AdS/CFT," arXiv:hep-th/0604100.

[29] G. Arutyunov, S. Frolov and M. Staudacher, "Bethe ansatz for quantum strings," JHEP 0410, 016 (2004) [arXiv:hep-th/0406256].

[30] N. Beisert and M. Staudacher, "Long-range PSU(2,2|4) Bethe ansaetze for gauge theory and strings," Nucl. Phys. B 727, 1 (2005) [arXiv:hep-th/0504190].

[31] D. Berenstein, D. H. Correa and S. E. Vazquez, "All loop BMN state energies from matrices," JHEP 0602, 048 (2006) [arXiv:hep-th/0509015].

[32] M. Kruczenski, "Spin chains and string theory," Phys. Rev. Lett. 93, 161602 (2004) [arXiv:hep-th/0311203].

[33] G. Arutyunov and S. Frolov, "Integrable Hamiltonian for classical strings on AdS(5) x S**5," JHEP 0502, 059 (2005) [arXiv:hep-th/0411089].

[34] M. Kruczenski, A. V. Ryzhov and A. A. Tseytlin, "Large spin limit of AdS(5) x $S^{* * 5}$ string theory and low energy expansion of ferromagnetic spin chains," Nucl. Phys. B 692, 3 (2004) [arXiv:hep-th/0403120].

[35] M. T. Grisaru, R. C. Myers and O. Tafjord, "SUSY and Goliath," JHEP 0008, 040 (2000) [arXiv:hep-th/0008015].

[36] A. Hashimoto, S. Hirano and N. Itzhaki, "Large branes in AdS and their field theory dual," JHEP 0008, 051 (2000) [arXiv:hep-th/0008016].

[37] J. Michelson, "(Twisted) toroidal compactification of pp-waves," Phys. Rev. D 66, 066002 (2002) [arXiv:hep-th/0203140].

[38] M. Bertolini, J. de Boer, T. Harmark, E. Imeroni and N. A. Obers, "Gauge theory description of compactified pp-waves," JHEP 0301, 016 (2003) [arXiv:hep-th/0209201]. 
[39] S. Frolov and A. A. Tseytlin, "Semiclassical quantization of rotating superstring in AdS(5) x S(5)," JHEP 0206, 007 (2002) [arXiv:hep-th/0204226].

[40] A. Dabholkar and S. Parvizi, "Dp branes in pp-wave background," Nucl. Phys. B 641, 223 (2002) [arXiv:hep-th/0203231].

[41] D. Berenstein, E. Gava, J. M. Maldacena, K. S. Narain and H. Nastase, "Open strings on plane waves and their Yang-Mills duals," arXiv:hep-th/0203249.

[42] A. A. Tseytlin, "Semiclassical strings and AdS/CFT," arXiv:hep-th/0409296.

[43] J. Cuntz, "Simple C* Algebras Generated By Isometries," Commun. Math. Phys. 57, 173 (1977).

[44] D. Minic, "Curiosities on free Fock spaces," arXiv:hep-th/9503203.

[45] D. Berenstein, "Large N BPS states and emergent quantum gravity," JHEP 0601, 125 (2006) [arXiv:hep-th/0507203].

[46] D. Berenstein and D. H. Correa, "Emergent geometry from q-deformations of $\mathrm{N}=4$ super Yang-Mills," arXiv:hep-th/0511104.

[47] J. P. Rodrigues, "Large N spectrum of two matrices in a harmonic potential and BMN energies," JHEP 0512, 043 (2005) [arXiv:hep-th/0510244].

[48] R. Hernandez and E. Lopez, "The SU(3) spin chain sigma model and string theory," JHEP 0404, 052 (2004) [arXiv:hep-th/0403139].

[49] S. Bellucci, P. Y. Casteill, J. F. Morales and C. Sochichiu, "sl(2) spin chain and spinning strings on AdS(5) x S**5," Nucl. Phys. B 707, 303 (2005) [arXiv:hep-th/0409086].

[50] S. A. Frolov, R. Roiban and A. A. Tseytlin, "Gauge - string duality for superconformal deformations of $\mathrm{N}=4$ super Yang-Mills theory," JHEP 0507, 045 (2005) [arXiv:hep-th/0503192].

[51] S. Benvenuti and M. Kruczenski, "Semiclassical strings in Sasaki-Einstein manifolds and long operators in $\mathrm{N}=1$ gauge theories," arXiv:hep-th/0505046.

[52] R. de Mello Koch, N. Ives, J. Smolic and M. Smolic, "Unstable giants," Phys. Rev. D 73, 064007 (2006) [arXiv:hep-th/0509007].

[53] W. M. Zhang, D. H. Feng and R. Gilmore, "Coherent States: Theory And Some Applications," Rev. Mod. Phys. 62 (1990) 867.

[54] S. S. Gubser, I. R. Klebanov and A. M. Polyakov, "A semi-classical limit of the gauge/string correspondence," Nucl. Phys. B 636, 99 (2002) [arXiv:hep-th/0204051].

[55] F. Calogero, "A solvable Hamiltonian system", J. Math. Phys. 36, 4832 (1995). 
[56] V. Karimipour, "Relation of The New Calogero Models and xxz Spin Chains," arXiv:hep-th/9603039.

[57] V. Karimipour, "A solvable Hamiltonian system: Integrability and action-angle variables," J. Math. Phys. 38, 1577 (1997) [arXiv:hep-th/9604092].

[58] A. R. Chowdhury and A. G. Choudhury, "Quantum Integrable Systems", Chapman \& Hall/CRC research notes in mathematics series, 435, (2004).

[59] H. Lin, O. Lunin and J. Maldacena, "Bubbling AdS space and 1/2 BPS geometries," JHEP 0410 (2004) 025 [arXiv:hep-th/0409174].

[60] N. Beisert, "The complete one-loop dilatation operator of $\mathrm{N}=4$ super Yang-Mills theory," Nucl. Phys. B 676, 3 (2004) [arXiv:hep-th/0307015].

[61] H. Takayanagi and T. Takayanagi, "Notes on giant gravitons on pp-waves," JHEP 0212, 018 (2002) [arXiv:hep-th/0209160].

[62] M. Arik and D. D. Coon, "Hilbert Spaces Of Analytic Functions And Generalized Coherent States," J. Math. Phys. 17, 524 (1976).

[63] M. El Baz and Y. Hassouni, "Deformed Exterior Algebra, Quons and Their Coherent States", Int. J. Mod. Phys. A 18, 3015 (2003). 